\title{
Tulane
}

Tulane Economics Working Paper Series

\section{Smoking, Expectations, and Health: A Dynamic Stochastic Model of Lifetime Smoking Behavior}

\author{
Michael Darden \\ Department of Economics \\ Tulane University \\ New Orleans, LA \\ mdarden1@tulane.edu \\ Working Paper 1204 \\ May 2012
}

\begin{abstract}
This research discusses results obtained through formulation and estimation of a dynamic stochastic model that captures individual smoking decision making, health expectations, and longevity over the life cycle. The standard rational addiction model is augmented with a Bayesian learning process about the health marker transition technology to evaluate the importance of personalized health information in the decision to smoke cigarettes. Additionally, the model is well positioned to assess how smoking, and smoking cessation, impacts morbidity and mortality outcomes while taking into consideration the potential for dynamic selection of smoking behaviors. The structural parameters are estimated using rich longitudinal health and smoking data from the Framingham Heart Study: Offspring Cohort. Results suggest that there exists heterogeneity across individuals in the pathways by which smoking effects health. Furthermore, upon smoking, the estimated parameters suggest a positive reinforcement effect and a negative withdrawal effect, both of which encourage future smoking. The paper also presents evidence of health selection in smoking behavior that, when not modeled, may cause an overstatement of the effect of smoking on morbidity and mortality. Finally, personalized health marker information is not found to significantly influence smoking behavior relative to chronic health shocks themselves.
\end{abstract}

Keywords: smoking, information, learning, structural econometrics JEL: I1, I12, D8, D83 


\title{
Smoking, Expectations, and Health: A Dynamic Stochastic Model of Lifetime Smoking Behavior
}

\author{
Michael Darden* \\ Tulane University $*$
}

October 12, 2011

\begin{abstract}
This research discusses results obtained through formulation and estimation of a dynamic stochastic model that captures individual smoking decision making, health expectations, and longevity over the life cycle. The standard rational addiction model is augmented with a Bayesian learning process about the health marker transition technology to evaluate the importance of personalized health information in the decision to smoke cigarettes. Additionally, the model is well positioned to assess how smoking, and smoking cessation, impacts morbidity and mortality outcomes while taking into consideration the potential for dynamic selection of smoking behaviors. The structural parameters are estimated using rich longitudinal health and smoking data from the Framingham Heart Study: Offspring Cohort. Results suggest that there exists heterogeneity across individuals in the pathways by which smoking effects health. Furthermore, upon smoking, the estimated parameters suggest a positive reinforcement effect and a negative withdrawal effect, both of which encourage future smoking. The paper also presents evidence of health selection in smoking behavior that, when not modeled, may cause an overstatement of the effect of smoking on morbidity and mortality. Finally, personalized health marker information is not found to significantly influence smoking behavior relative to chronic health shocks themselves.
\end{abstract}

\footnotetext{
*Email:mdarden1@tulane.edu

The Framingham Offspring Study (FOS) is conducted and supported by the NHLBI in collaboration with the FOS Study Investigators. This manuscript was prepared using a limited access dataset obtained from the NHLBI and does not necessarily reflect the opinions or views of the FOS or the NHLBI.

Department of Economics, 206 Tilton Memorial Hall, Tulane University, New Orleans, LA 70118
} 


\section{Introduction}

The decision to smoke has long interested social scientists and health policy researchers because of the seemingly irrational nature of such a choice. Why would an individual undertake an activity with such clear negative health consequences? A thorough review of this debate can be found in Sloan et al. (2003). Ultimately, those authors conclude that individuals make decisions within an environment that reflects individual preferences but one that is also subject to information acquisition costs. Gary Becker describes economic decision makers: "They (economic agents) are not expected to be perfect optimizers, as evaluated by the analyst, or dispassionate external observers; rather, people do the best they can, given their information and their cognitive abilities to understand it (qtd. in Sloan et al. (2003) pg. 25)." An important question addressed by the smoking literature has been: what determines and shapes "their information?" Furthermore, how does information influence smoking behavior? And, to what extent has information regarding the health effects of smoking been free from selection bias? These questions form the basis for the current paper.

To address these questions, this paper estimates the structural parameters of an individual's optimization problem with the following trade-off: current enjoyment of cigarette consumption versus the associated and uncertain future health, and thus, utility consequences. Two dimensions of health are considered: health markers and chronic health. Health markers are those factors (e.g., blood pressure, cholesterol, etc.) viewed by the medical literature to significantly predict the onset of chronic conditions (e.g., cardiovascular disease, cancer, etc.). In this setting, health markers are framed as informative signals of the health effects of cigarette smoking. Given a history of these health markers and smoking behaviors, an individual is able to more precisely evaluate the effect of smoking on her health markers levels which, in turn, helps to determine her chronic health probability. Endowed with this information, an individual makes the smoking choice that maximizes her present discounted expected utility.

The structural parameters of the model are estimated with rich longitudinal data from the offspring of the original cohort of the Framingham Heart Study. These data record health examinations over a 30 year period and, due to wide age variation, capture health and smoking outcomes across the life cycle. The structural model is identified from variation in the timing of health exams. That is, observationally equivalent individuals that receive health exams at different time intervals, form different beliefs and may make different smoking decisions; however, the timing of Framingham Heart Study exams was not related to health.

This paper furthers our understanding of smoking dynamics in three important ways. First, the structural model extends the standard rational addiction model of Becker and Murphy (1988) by incorporating both health and learning. While other papers that incorporate 
health and/or learning into the rational addiction framework are purely theoretical (e.g., Orphanides and Zervos (1995); Suranovic et al. (1999); Carbone et al. (2005)), I estimate the preference and expectation parameters of a rational addiction model that capture forwardlooking behavior with respect to health. ${ }^{1}$ Smoking history is modeled as a capital stock and is measured in a novel way so as to facilitate the estimation of depreciation and investment coefficients while keeping the model computationally tractable. Consistent with the theory of rational addiction, I find that smoking is reinforcing in the sense that the marginal utility of smoking is increasing in the amount of past smoking. I also find that the costs of withdrawal can prevent individuals from quitting smoking. The reinforcement and withdrawal effects are found to drive smoking dynamics.

Second, a major contribution of this paper is to model how the receipt of information about an individual's health markers may alter an individual's smoking behavior. When information about the ill-health effects of smoking is made personal, the literature has argued that this personalized health information may provide an individual with a powerful incentive or "wake-up call" to curtail smoking behavior (Sloan et al., 2003). ${ }^{2}$ As an example, if a smoker experiences a heart attack, this health shock may be framed as an informative signal of the consequences of her smoking. This paper extends the literature on smoking responses to personalized health information that have only considered chronic health shocks (e.g., heart attacks, cancer diagnoses, etc.) (Smith et al., 2001; Khwaja et al., 2006; Arcidiacono et al., 2007). The distinction between health marker information and chronic health shocks as information is important if the potential gains from information from a chronic health shock are "too late". Indeed, if policy makers are concerned with improving overall expected longevity, the incidence of a heart attack, while effective in convincing an individual to quit smoking, may not yield additional longevity. However, if changes in blood pressure, say, over time that are due to smoking, convince an individual to quit prior to the incidence of a heart attack, then there may exist a role for policy to emphasize the personalized "warning signs." Thus, motivated by the Becker quote above, the structural model in this paper explicitly accounts for health marker transition learning, and estimates parameters that dictate a Bayesian learning process.

To evaluate the roles of learning and information, I use the model and the estimated

\footnotetext{
${ }^{1}$ Individuals are modeled as forward-looking in the sense that they evaluate current smoking alternatives while taking into consideration the future health and utility consequences associated with past and current smoking behavior. See Viscusi (1990); Viscusi and Hakes (2008); Arcidiacono et al. (2007) for evidence that smokers are forward-looking with respect to health.

${ }^{2}$ Specifically, Sloan et al. (2003) and Khwaja et al. (2006) argue that, while general sources of health information (e.g., commercials, warning labels, etc.) may influence the light to moderate smoker, these sources of information will have less affect on the smoking behavior of heavy smokers. Indeed, for the heavy smoker, personalized information is required to change behavior.
} 
structural parameters to simulate smoking behavior and health and mortality outcomes under different counterfactual scenarios. The results suggest that there exists heterogeneity across individuals in the pathways by which smoking affects health. I find that the effect of the accumulated smoking stock on health markers varies widely across individuals relative to the mean effect. While the average variance in beliefs regarding this effect decreases by $20 \%$ after the first health exam, the estimated mean of the parameter distribution is small and thus, learning about this parameter does not greatly impact smoking behavior. Counterfactuals in which individuals receive information more frequently do not appear to show individuals altering their smoking behavior in any significant sense. However, when signals of poor health markers are augmented between two and four times their normal levels, individuals are simulated to quit smoking at the ages in which the major chronic smoking-related shocks start to occur. While smokers are found to quit or reduce their smoking after a chronic health shock, results from this paper suggest that health markers have limited informational value.

Tangentially, a third contribution of this paper brings into question whether current knowledge of the health effects of smoking are free from selection bias. Using data from across the life cycle, this paper measures the role of health and mortality transition determinants by estimating these production technologies within the structural model of lifetime smoking decisions. This method allows both for smoking to affect health and for rational individuals to select the optimal smoking alternative while taking into account their health. Modeling both smoking behavior and health outcomes also allows for the capture of unobserved heterogeneity that may be present in their joint determination. For example, if smokers are also more likely to engage in other health-hazardous behaviors (e.g. drinking, drug use, etc.), then treating smoking as random within the population will lead to overstated estimates of the direct effect of smoking on the health outcome of interest.

My results are the first of which I am aware to explicitly control for the potential positive selection between smoking and health outcomes while also estimating the health transition equations within the structural model of lifetime smoking. To capture any unobserved heterogeneity, I allow the unobserved errors that affect smoking, health, and mortality to be serially correlated through a common permanent unobserved component. I model this error structure with the discrete factor method (Heckman and Singer, 1984; Mroz, 1999) which amounts to a random effects specification of unobserved heterogeneity that is free from distributional assumptions. $^{3}$

\footnotetext{
${ }^{3}$ The error structure is similar to recent structural models that have accounted for unobserved heterogeneity (Arcidiacono et al., 2007; Blau and Gilleskie, 2008).
} 
To assess both the potential for selection on smoking and the effect of smoking on health outcomes, I simulate the structural model under different lifetime smoking patterns and heterogeneity characteristics. For daily light and heavy smoking from age 18, individuals can expect roughly 4.5 and 8 fewer years of longevity, respectively. These results are less severe in their overall assessment of the health effects of smoking on mortality than are the unconditional results presented in the widely cited work of Doll et al. $(1994,2004)$. My results indicate that there exists a strong positive correlation between smoking tendencies and underlying factors that influence mortality outcomes that, if ignored, leads to an overstatement of the longevity effects of cigarette smoking. Interestingly, while I find that unobserved heterogeneity plays a major role in the dynamic relationship between smoking behavior and mortality, the unobserved heterogeneity plays almost no role in predicting health marker and chronic health transitions.

Still, consistent with some literature on smoking and health, my results suggest that there exist longevity gains from quitting at any age, and that quitting prior to age 30 implies that an individual has roughly the same expected longevity as a lifelong nonsmoker. Furthermore, this paper finds that quitting heavy smoking at ages 30,40, 50, and 60 years of age increases life-expectancy by approximately $8,7.75,7$, and 5.5 years, respectively. ${ }^{4}$

Finally, another contribution of this paper is the way in which smoking dynamics are modeled empirically. The key term in the standard rational addiction model of Becker and Murphy (1988) is an addictive capital stock that is subject to investment and depreciation. Those authors argue that addiction is captured by a positive interaction between the smoking stock and the marginal utility of addictive consumption. While theoretically it is quite intuitive to think an individual that has smoked in the past may have a higher marginal utility of smoking than someone who has not smoked, it is not clear how to best capture this capital stock empirically. For example, defining the stock to be the total number of years smoked ignores the importance of cessation (i.e., someone who smoked for 10 years and quit twenty years ago may have a different marginal utility for smoking than someone who smoked for 10 years and quit last year). This paper extends the empirical smoking literature with a novel construction of the "smoking stock". Using factor analysis in a method similar to Sickles and Williams (2008), I create a continuous smoking stock index from several variables that reflect

\footnotetext{
${ }^{4}$ There is a large literature on the effects of smoking cessation on health outcomes. See United States Department of Health and Human Services (1990); Doll et al. (1994); Taylor et al. (2002); Doll et al. (2004); Brønnum-Hansen et al. (2007). This issue of "smoking depreciation" is often framed as the amount of time required after cessation until the probability of a health outcome converges to that of a nonsmoker. Because of the generality of my treatment of health (e.g., chronic health or not), and because smoking cessation improves various health outcome probabilities differently, the discussion in this paper focuses on the benefits from quitting smoking on expected longevity.
} 
past smoking behavior. ${ }^{5}$ This easily interpretable state variable captures the unique smoking history that each individual brings into each decision making period. Furthermore, measuring the smoking stock using this method also allows for the estimation of depreciation and investment parameters. ${ }^{6}$

This paper proceeds in the following sections. Section I provides background on the economics and medical literatures with respect to smoking. That section also describes the economics of information and expectations in the context of cigarette smoking. Section II presents the formal structural model. Section III discusses the Framingham Heart Study and presents basic summary statistics of the data used in estimation. How the structural model in Section II and the data in Section III are reconciled is discussed in Section IV. I also formally describe the econometric methods used in estimating the structural model as well as identification issues in Section IV. Section V presents the main results of the paper and examines potential policy measures. Section VI offers a brief discussion and concludes. Additionally, Appendix A provides details on the solution method employed in this paper, and Appendix B gives the formal derivation of posterior beliefs.

\section{Background}

Cigarette smoking is the single greatest preventable risk factor for mortality and morbidity. According to a 2004 Surgeon General report, cigarette smoking is causally linked to cancers of the bladder, cervix, esophagus, kidney, larynx, lung, mouth, pancreas, and stomach. Furthermore, there exists a causal relationship between smoking and coronary heart disease, cerebrovascular disease, atherosclerosis, various respiratory diseases, and several reproductive maladies. 440,000 deaths are attributed to smoking in the United States each year. Illness from smoking is estimated to add $\$ 157$ billion per year to national health expenditures. In short, a 2004 United States Surgeon General report on smoking concludes by stating: "Smoking harms nearly every organ of the body, causing many diseases and reducing the health of smokers in general."7

Behind much of the Surgeon General Report's results is the ongoing work of (Doll et al., 1994, 2004). Those authors use survey data of British physicians over several decades to assess

\footnotetext{
${ }^{5}$ Sickles and Williams (2008) use principal component analysis to construct a "stock" of social capital from several correlated variables such as labor force participation, yearly hours worked, marriage, and "hours in income generating crime per year."

${ }^{6}$ Adda and Lechene (2004) note that the smoking stock may be different between reinforcing further smoking and affecting health. Indeed, those authors model two smoking stocks: a utility smoking stock and a health smoking stock. Each are subject to different investment and depreciation parameters; however, those authors fix rather than estimate these parameters. In my case, I abstract from this possibility and estimate the transition parameters of a single stock variable that captures past smoking.

${ }^{7}$ Centers for Disease Control and Prevention, 2004. http://www.cdc.gov/tobacco/basic_information/index.htm
} 
the impact of cigarette smoking on mortality. Their findings suggest that smoking cessation at ages 30, 40, 50, and 60 lead to improved life expectancies of 10, 9, 6, and 3 years respectively. Furthermore, life-long smokers face a roughly 25 percentage point increase in the probability of death during middle aged (35-69). However, Doll et al. do not control for the endogeneity of smoking with respect to health outcomes nor do they consider the possibility that doctors in different health states may select into smoking. If, independently of smoking, smokers are of a worse overall health status than non-smokers, standard statistical methods may overstate the effect of smoking on mortality. Indeed, only recently have papers in health economics begun to jointly model smoking and health outcomes. For example, Adda and Lechene (2001) show that potential life-span and smoking behavior are correlated along unobserved (to the econometrician) dimensions. In the current paper, estimation of the primitive parameters of one's decision making optimization problem (e.g., preferences, constraints, and expectation parameters) allows me to assess the impact of smoking on morbidity and mortality outcomes while considering the potential for endogeneity of and dynamic selection into smoking behaviors. In addition, the introduction of serially correlated (permanent) unobserved heterogeneity that affects decision making over the life cycle (including observed initial conditions) allows for the recovery of parameters that measure the impact of smoking and health markers on morbidity and mortality that are free of selection bias. Unbiased estimation of these primitive parameters allows me to simulate the model and impose different patterns of smoking and quitting to examine the resulting changes in predicted health outcomes.

In addition to assessing the potential for dynamic selection with respect to smoking and health outcomes, the main goal of this paper is to assess the importance of health learning within the rational addiction framework of Becker and Murphy (1988). One branch of the rational addiction literature has studied the roles of information, risk perceptions, subjective expectations, and learning in the decision to smoke. Viscusi (1990) models an individual's beliefs regarding her health risk from cigarette smoking as a Bayesian function of three factors: a prior risk assessment, some measure of risk from experience (perhaps smoking history, age, etc.), and some new information regarding risk. An important question addressed by the literature has been: what exactly is this new risk information?

One type of new information can be categorized as any information that is directed toward a general audience. A widely publicized example was the landmark 1964 United States Surgeon General report that linked smoking to lung cancer and certain birth defects. Luther L. Terry, then Surgeon General, stated that the report "hit the country like a bombshell. It was front page news and a lead story on every radio and television station in the United States."

\footnotetext{
${ }^{8}$ http://profiles.nlm.nih.gov/NN/Views/Exhibit/narrative/smoking.html
} 
Did this information deter individuals from taking up smoking? Did smokers at the time respond to the report by quitting? On this question, the literature has been mixed. While much of the literature suggests that informational anti-smoking campaigns decrease cigarette demand for light to moderate smokers, Sloan et al. (2003) argue that heavy smokers "do not appear to update these perceptions (on the probability of illness/death due to smoking) in response to general information; they need the message to be personalized."

Personalized health information may be an important motivator to quit if heavy smokers possess an "it won't happen to me" attitude. Khwaja et al. (2006), studying individuals from the Health and Retirement Survey (HRS), show that smokers only "learn" about the risks associated with smoking, as measured by a change in smoking behavior, from a shock to their own health. Those authors argue that if any health shock other than one's own would encourage smoking cessation, it should be that of a spouse. The authors however find no significant effect of spousal health shocks on smoking behavior. "The clear differences in the effects of smoking-related health shocks for current smokers suggest that personalized messages, relevant to their circumstances, are necessary to get their attention and induce changes in their beliefs (qtd. in Sloan et al. (2003) pg. 124)."

Nearly all previous work that has examined learning or expectation formation with respect to personalized health messages has studied behavioral changes after a major health shock to self or spouse (Smith et al., 2001; Khwaja et al., 2006; Arcidiacono et al., 2007). Additionally, most papers focus on individuals above the age of 50, at which age we begin to observe the major health implications of smoking. However, waiting for a major health shock to incite individuals to quit smoking may be too late in terms of life expectancy gains. Therefore, the current paper examines the extent to which individuals learn about smoking and health from personalized health marker information.

Using Framingham Heart Study data, Garrison et al. (1978) shows that cigarette smoking has a negative impact on high-density lipoprotein (HDL) cholesterol, or "good" cholesterol. Furthermore, due to the nicotine content in cigarettes, other studies have shown that smoking increases both heart rate and blood pressure (Bennett and Richardson, 1984; Omvik, 1996). These health markers, among others, have been directly tied to the risk of cardiovascular disease. To my knowledge, no study has examined the impact of personalized health marker information on the decision to smoke.

Methodologically, this paper builds on other structural models that estimate Bayesian learning processes in pharmaceutical demand (Crawford and Shum, 2005; Chan and Hamilton, 2006), fertility and infant mortality (Mira, 2007), marketing (Ackerberg, 2003), and health plan report cards (Chernew et al., 2008). While currently beyond the scope of this

\footnotetext{
${ }^{9}$ Italics theirs.
} 
paper, interesting future work might also consider other preference specifications such as nonexpected utility (e.g., Kreps and Porteus (1978, 1979); Epstein and Zin (1991)), hyperbolic preferences (e.g., Gruber and Koszegi (2001)), and cue-trigger mistakes (e.g., Bernheim and Rangel (2004)) in the context of smoking and addiction.

\section{Theoretical Model}

I specify a dynamic stochastic model of smoking behavior that incorporates learning. This section outlines the basic theoretical model. Given the limitations of the data, changes to the model in the empirical implementation are discussed in section IV. Furthermore, the appendices provide derivations and details of my solution method.

Consider a mixed discrete/continuous-state, discrete-time model of smoking behavior. The model has a finite horizon in the sense that, while an individual may die prior to period $T$, the probability of death equals one in period $T$. A period is indexed by subscript $t$ and is assumed to be one year in length. Each period, a forward-looking individual makes a smoking decision to maximize her lifetime discounted expected utility. Let the decision for individual $i$ be given by $d_{i t}=d$, where smoking alternative $d$ is:

$$
d=\left\{\begin{array}{ll}
0 & \text { Do not smoke } \\
1 & \text { Smoke } \leq 1 \text { Pack/day } \\
2 & \text { Smoke }>1 \text { Pack/day }
\end{array}\right\}
$$

The set of factors that influence individual $i^{\prime} s$ smoking decision in period $t$ are given by the state space $S_{i t}$. Define $S_{i t}$ as follows:

$$
S_{i t}=\left\{A_{i t}, R_{i t}, \tau_{i t}, \psi_{i t}, H_{i t}, X_{i t}\right\}
$$

where $A_{i t}$ is individual $i^{\prime} s$ smoking stock entering period $t$; $R_{i t}$ is an index of her health markers; $\tau_{i t}$ and $\psi_{i t}$ are her mean and variance respectively of her posterior belief distribution; $H_{i t}$ is her chronic health status; and $X_{i t}$ is her set of demographic characteristics. Additionally influencing behavior, but not listed here, are a preference error $\epsilon_{i t}$ and a permanent heterogeneity term $\mu$ that are both assumed to be known to the individual but unobserved to the econometrician. Assumptions about these error terms that aid estimation are discussed in section IV.

At the beginning of representative period $t$, an individual undergoes her period $t$ health exam and realizes her period $t$ chronic health state $H_{i t}$, health marker index $R_{i t}$, and smoking stock $A_{i t}$. Using the information from the period $t$ health exam, an individual then updates her 
beliefs regarding the evolution of future state variables. The smoking decision is then made and utility (to be defined below), as a function of the decision and period $t$ state variables, is realized.

In the subsections below, I expand upon each of the observed state variables and preferences.

\section{II.1 Smoking Stock}

Following the rational addiction literature, define $A_{i t}$ as the accumulated smoking stock. ${ }^{10}$ Formally, define the stock as:

$$
A_{i t}=\left\{\begin{array}{ll}
\exp \left\{\delta_{1} \ln \left(A_{i t-1}\right)+\delta_{2} \mathbf{1}\left[d_{i t-1}=1\right]+\delta_{3} \mathbf{1}\left[d_{i t-1}=2\right]+\rho^{A} \mu+\eta_{i t}\right\} & \text { if } \begin{array}{l}
\sum_{n=1}^{t-1} d_{i n}>0 \\
0
\end{array}
\end{array}\right\}
$$

Equation 1 says that individual $i$ 's time $t$ smoking stock is normalized to 0 if she has not smoked in any previous period. Conditional on any past smoking, the stock is specified as a function of the previous period stock and the previous period decision. $\delta_{1}$ can be interpreted as one minus the depreciation rate of the stock in percentage terms. The nonlinear investment of light and heavy smoking into the smoking stock are captured by $\delta_{2}$ and $\delta_{3}$, respectively. Unobserved permanent heterogeneity is captured by the $\mu$ term and its factor loading $\rho^{A} .{ }^{11}$ Also influencing the stock is an i.i.d. white noise term, $\eta_{i t}$, which is distributed $\mathscr{N}\left(0, \sigma_{\eta}\right){ }^{12}$ Consistent with the interpretation of the stock as a summary of an individual's smoking history, the stock is assumed to be known by the individual in each period.

\section{II.2 Health Marker Index}

Define $R_{i t}$ as a continuous scalar summary of a variety of health markers (e.g., blood pressure, cholesterol, etc.) that is realized by the individual in each period. Similar to the smoking stock $\left(A_{i t}\right), R_{i t}$ is a scalar representation of numerous health factors. I assume that $R_{i t}$ evolves as follows:

$$
R_{i t}=\zeta R_{i t-1}+X_{i t} \phi+\kappa_{i t}+\rho^{R} \mu .
$$

\footnotetext{
${ }^{10}$ The concept of a smoking "stock" is not immediately intuitive. Broadly speaking, the rational addiction literature treats the stock as a measure of past smoking. Medically however, we might consider the stock as some accumulation of tar in the lungs that influences health. Alternatively, we might think of the stock as the extent of dependence on nicotine. For the purposes of this paper, the stock may be interpreted as a continuous summary of an individual's smoking history. Here, the extent to which $A_{i t}$ influences health is an empirical question. See the empirical section for further discussion.

${ }^{11}$ See section IV for a discussion of estimation and interpretation issues regarding the permanent unobserved heterogeneity.

${ }^{12}$ Given the exponential stock evolution equation, $\eta$ is a log normal shock.
} 
Here, $X_{i t}$ is a vector of sociodemographic characteristics of individual $i$. I assume that the technology associated with these characteristics (i.e., $\phi$ ) is known by the individual. $\zeta$ captures the dynamic aspect of the health markers and is also assumed to be known to the individual. Time invariant and unobserved (to the econometrician) heterogeneity is captured by the $\mu$ term and its factor loading $\rho^{R}$. Let $\kappa_{i t}$ represent the input from the smoking stock plus an idiosyncratic, i.i.d. error term that is defined as:

$$
\kappa_{i t}=\theta_{i} A_{i t}+v_{i t} .
$$

Because the individual observes or knows $R_{i t}, \zeta, X_{i t} \phi$, and $\rho^{R} \mu, \kappa_{i t}$ is also observed by the individual. The medical literature suggests that there exists heterogeneity across individuals in the health effects of smoking. I theorize that each individual is endowed with a time invariant, unknown (to both the individual and econometrician) match value $\theta_{i}$ that captures the idiosyncratic effect of the smoking stock, $A_{i t}$ on the health marker index, $R_{i t}$. I assume that $\theta_{i}$ is drawn from a known population distribution given by:

$$
\theta_{i} \sim \mathscr{N}\left(\bar{\theta}, \sigma_{\theta}^{2}\right) .
$$

$\kappa_{i t}$ therefore serves as an informative signal. Over time, by having health exams and thus observing a sequence of signals, $\theta_{i}$ is learned in a Bayesian fashion. Learning is, however, confounded by the i.i.d. noise term, $v_{i t}$. Indeed, without $v_{i t}$, an individual would perfectly learn their match value $\theta_{i}$ at the first health exam (i.e., the first realization of $\kappa_{i t}$ ). While $v_{i t}$ is unknown, its distribution is known and given by:

$$
v_{i t} \sim \mathscr{N}\left(0, \sigma_{v}^{2}\right) .
$$

Because $\theta_{i}$ is time invariant, and because the distributions of $\theta_{i}$ and $v_{i t}$, as well as the stock $A_{i t}$, are known, over time, an individual can learn their idiosyncratic value of $\theta_{i} \cdot{ }^{13}$

\section{II.3 Learning}

Let an individual's period $t$ posterior beliefs, those with which she forecasts future health markers, be given by $\tau_{i t}$, her posterior mean, and $\psi_{i t}$, her posterior variance. I assume rational expectations such that an individual's initial belief, prior to any health exams, regarding her true $\theta_{i}$ (the marginal effect of one's smoking history, $A_{i t}$, on health markers, $R_{i t}$ ) is the population distribution. ${ }^{14}$ Initial beliefs $(t=0)$ are:

$$
\tau_{i 0}=E_{0}\left(\theta_{i}\right)=\bar{\theta}
$$

\footnotetext{
${ }^{13}$ The assumption that an individual knows the technology of the health production function is ubiquitous in health economics. That is, typically $\theta_{i}=\bar{\theta} \forall i$ and $\sigma_{\theta}=0 . \bar{\theta}$ is then estimated and assumed to be the marginal product that all individuals use to solve optimization problems.

${ }^{14}$ The rational expectations assumption is what is typically made in most models of health transitions.
} 


$$
\psi_{i 0}=V_{0}\left(\theta_{i}\right)=\sigma_{\theta}^{2} .
$$

Expectations about future health marker transitions evolve in the current model with the receipt of personalized health information. In deriving posterior beliefs, consider an individual in period $t$ with smoking stock $A_{i t}$. This individual has two fundamental sources of information: her prior beliefs, $\left(\tau_{i t-1}, \psi_{i t-1}\right)$, and the observed results from her period $t$ health exam, $\kappa_{i t}$. Appealing to the assumption of conjugate prior and signal distributions, the period $t$ beliefs have closed form solutions that are given via Bayes' Rule. The posterior mean and variance are $:^{15}$

$$
\begin{gathered}
\tau_{i t}=E\left(\theta_{i} \mid \kappa_{i t}, A_{i t}, \tau_{i t-1}, \psi_{i t-1}\right)=\frac{A_{i t}^{2} \psi_{i t}}{\sigma_{v}^{2}} \hat{\theta}_{i t}+\frac{\psi_{i t}}{\psi_{i t-1}} \tau_{i t-1} \\
\psi_{i t}=\operatorname{Var}\left(\theta_{i} \mid A_{i t}, \psi_{i t-1}, \sigma_{v}\right)=\frac{\psi_{i t-1} \sigma_{v}^{2}}{A_{i t}^{2} \psi_{i t-1}+\sigma_{v}^{2}} .
\end{gathered}
$$

Here, $\hat{\theta}_{i t}$ is the least squares estimate of $\kappa_{i t}$ on $A_{i t}$ from the within individual variation of the $t^{t h}$ health exam. Note that these beliefs have the following appealing properties. First, the posterior mean is a weighted average of $\hat{\theta}_{i t}$ and the original prior mean $\tau_{i t-1}$. Second, the weight placed on the period $t$ signal (i.e., $\hat{\theta}_{i t}$ ) is increasing in the smoking stock. Finally, the posterior moments of an individual for whom the stock equals zero (i.e., $A_{i t}=0$ ) collapse to the prior moments.

\section{II.4 Chronic Health}

Let $H_{i t}$ represent an individual's overall health state. An individual's overall health state is determined by the presence of any chronic conditions. Let $H_{i t}=h$, where outcome $h$ is as follows:

$$
h=\left\{\begin{array}{lll}
1 & \text { if } & \text { Chronic Condition } \\
0 & \text { if } & \text { No Chronic Condition }
\end{array}\right\}
$$

What differentiates $H_{i t}$ and $R_{i t}$ is "reversibility". While $R_{i t}$ changes each period, I assume that upon diagnosis of a chronic condition, an individual has the condition forever. ${ }^{16}$ Let the probabilities of transiting to different chronic health states in period $t+1$ be:

$$
\begin{gathered}
\pi_{i t+1}^{h=0}=\left\{\begin{array}{ll}
{\left[1-P\left(H_{i t+1}=1 \mid S_{i t}, d_{i t}, \mu\right)\right]} & \text { if } H_{i t}=0 \\
0 & \text { if } H_{i t}=1
\end{array}\right\} \\
\pi_{i t+1}^{h=1}=\left\{\begin{array}{ll}
P\left(H_{i t+1}=1 \mid S_{i t}, d_{i t}, \mu\right) & \text { if } H_{i t}=0 \\
1 & \text { if } H_{i t}=1
\end{array}\right\} .
\end{gathered}
$$

\footnotetext{
${ }^{15}$ Derivations of these equations can be found in Appendix B.

${ }^{16}$ This assumption captures the fact that upon having an heart attack, for example, an individual is in a fundamentally different health state even if they don't have repeated heart attacks (Khwaja et al., 2006).
} 
Define the relevant probability, $P\left(H_{i t+1}=1 \mid S_{i t}, d_{i t}, \mu\right)$, with the following binary logit equation:

$$
\frac{\exp \left(\lambda_{0}+\lambda_{1} R_{i t}+\lambda_{2} R_{i t}^{2}+\lambda_{3} 1[1980 s] * R_{i t}+\lambda_{4} 1[1990 s] * R_{i t}+\left[\lambda_{5}+\lambda_{6} R_{i t}\right] * d_{i t}+\lambda_{7} X_{i t}+\rho^{H} \mu\right)}{1+\exp \left(\lambda_{0}+\lambda_{1} R_{i t}+\lambda_{2} R_{i t}^{2}+\lambda_{3} 1[1980 s] * R_{i t}+\lambda_{4} 1[1990 s] * R_{i t}+\left[\lambda_{5}+\lambda_{6} R_{i t}\right] * d_{i t}+\lambda_{7} X_{i t}+\rho^{H} \mu\right)}
$$

Here, $R_{i t}$ is the health marker index defined above, $X_{i t}$ is a vector of exogenous individual characteristics, $d_{i t}$ is the smoking choice and $\mu$ is an individual, time invariant unobserved heterogeneity term. The factor loading superscript $H$ simply differentiates it from other factor loadings in the model. $\lambda_{6}$ and $\lambda_{7}$ capture changes over time in how health markers affect the probability of chronic disease incidence (perhaps due to advances in medical technology, pharmaceuticals, etc.). In forecasting future chronic health transitions, I follow the literature and assume that an individual has rational expectations and that she understands the technology associated with the chronic health transition probability.

A natural question becomes, why do individuals in the model learn about how smoking affects health markers but not chronic conditions? By modeling learning about the effect of $A_{i t}$ on $R_{i t}$, however, individuals are indirectly updating their expectations about future chronic health transitions because the health marker index enters the chronic health transition probability. Furthermore, the purpose of this paper is to explore the importance of health information prior to major health shocks. Imposing that individuals understand the technology (i.e., the $\lambda \mathrm{s}$ ) associated with covariates in the chronic health transition equation is the standard approach. While future work may incorporate learning about health transition probabilities, such learning is currently beyond the scope of this paper.

\section{II.5 Mortality}

While an individual may die prior to period $T$, death is assumed to occur with probability one in period T. Define an indicator for death at the end of period $t, M_{i t+1}=1$, and let its corresponding probability, $\varsigma_{i t+1}=P\left(M_{i t+1}=1 \mid S_{i t}, d_{i t}, \mu\right)$, be given by:

$$
\frac{\exp \left(\omega_{0}+\omega_{1} R_{i t}+\omega_{2} R_{i t}^{2}+\omega_{3} H_{i t+1}+\left[\omega_{4}+\omega_{5} R_{i t}+\omega_{6} H_{i t+1}\right] * d_{i t}+\omega_{7} 1[1980 s] * H_{i t+1}+\omega_{8} 1[1990 s] * H_{i t+1}+\omega_{9} X_{i t}+\rho^{M} \mu\right)}{1+\exp \left(\omega_{0}+\omega_{1} R_{i t}+\omega_{2} R_{i t}^{2}+\omega_{3} H_{i t+1}+\left[\omega_{4}+\omega_{5} R_{i t}+\omega_{6} H_{i t+1}\right] * d_{i t}+\omega_{7} 1[1980 s] * H_{i t+1}+\omega_{8} 1[1990 s] * H_{i t+1}+\omega_{9} X_{i t}+\rho^{M} \mu\right)}
$$

Here, $H_{i t+1}$, is individual $i^{\prime} s$ chronic health state at the end of period $t .{ }^{17}$ Again, the superscript on the factor loading simply differentiates it from other factor loadings. The technology for the death transition equation is assumed to be known by the individual. $\omega_{8}$ and $\omega_{9}$ capture the fact that, conditional on having some chronic illness, the probability of death from that illness may have changed over time due to medical advances. Furthering the discussion above,

\footnotetext{
${ }^{17}$ The timing convention here is due to data aggregation. Clearly, any chronic health event occurring in period $t$ must occur at or before the time of death, if death also occurs in $t$. Therefore, to accommodate the frequent observation in the data of an individual dying from a chronic health event, the appropriate chronic health data point in this equation is $H_{i t+1}$.
} 
because the health marker index enters the death transition equation directly (and indirectly through the chronic health term $H_{i t}$ ), individuals are indirectly updating their expectations about death transitions conditional on their smoking choice through the learning process.

\section{II.6 Preferences}

Following the standard expected utility framework, the deterministic portion of per period utility associated with health state $h,(h=0,1)$, and smoking alternative $d_{i t}=d$ is:

$$
\begin{aligned}
\bar{U}_{i t}^{h}\left(A_{i t}, d_{i t}=d, R_{i t}, X_{i t}, \mu\right)= & \alpha_{0 h}+\left(\alpha_{1 h}+\alpha_{2 h} A_{i t}+\alpha_{3 h} R_{i t}+\alpha_{4 h} A g e_{i t}\right) *\left[d_{i t}=1\right] \\
& +\left(\alpha_{5 h}+\alpha_{6 h} A_{i t}+\alpha_{7 h} R_{i t}+\alpha_{8 h} A g e_{i t}\right) *\left[d_{i t}=2\right] \\
& +\alpha_{9 h} *\left[d_{i t-1} \neq 0\right] *\left[d_{i t}=0\right]+\alpha_{10 h} A_{i t}+\alpha_{11 h} A_{i t}^{2}+\rho^{\text {Uhd }} \mu
\end{aligned}
$$

The specification accommodates any nonlinearity in the effects of light and heavy smoking on utility. While $\alpha_{1}$. $\left(\alpha_{5 .}\right.$ ) is the direct marginal utility of light (heavy) smoking, $\alpha_{2 .}\left(\alpha_{6 .}\right.$ ) captures the extent to which past consumption reinforces current consumption. $\alpha_{2}$. $\left(\alpha_{6}\right.$.) captures a part of the intertemporal trade-off in utilities. The extent to which the health marker index affects the marginal utility of smoking is captured by $\alpha_{3} .\left(\alpha_{7}\right)$. Note that higher values of $R_{i t}$ and $A_{i t}$ imply worse health and a higher smoking stock respectively. The sign and magnitudes of $\alpha_{2}$. $\alpha_{3 .}, \alpha_{6}$, and $\alpha_{7}$. are empirical questions. $\alpha_{4}$. $\left(\alpha_{8}\right.$.) captures changes in the marginal utility of smoking across the lifespan. Specific withdrawal costs from quitting, which also capture part of the intertemporal utility trade-off, are captured by $\alpha_{9}$. Finally, $\alpha_{10}$. and $\alpha_{11}$. capture tolerance in smoking. That is, the extent to which a given level of stock affects utility is captured here regardless of smoking behavior. ${ }^{18}$

Relative preferences over smoking alternatives hinge on two main factors. First, preferences vary by the chronic health state $\left(H_{i t}=h\right)$. The extent to which the marginal utility of smoking varies across chronic health states remains an open question. Generally, the marginal utility of consumption of any normal good is thought to be lower in worse health states (Viscusi and Evans, 1990; Gilleskie, 1998). If however smoking provides relaxation and comfort when stricken with a chronic illness, the overall marginal utility of smoking may be larger in worse health states. Estimation of the structural parameters will therefore empirically test for the sign of the marginal utility of smoking across health states. Second, as seen in equations

\footnotetext{
${ }^{18}$ I have considered alternative specifications in which decade dummies shift the marginal utility of each smoking alternative to capture the changes in national trends and attitudes towards cigarette smoking. However, such a specification assumes that individuals have perfect foresight on future attitudes towards smoking. Also considered were nonlinear age terms to capture youth, middle-age, and elderly marginal utility shifts; however, these thresholds would be arbitrarily chosen and the computational problem with adding additional parameters is large.
} 
1,2 , and 3, current period smoking affects the size of the next period smoking stock, which in turn affects the next period health marker index and next period utility. Given the dynamic nature of the model, individuals evaluate smoking alternatives while considering the future marginal utility of smoking as well as the future consequences of a higher $A_{i t}$.

Following Rust (1987), let the total current period utility be the sum of the deterministic utility from equation 8 and an additive i.i.d. preference shock that is alternative and health-state specific:

$$
U_{i t}^{h}\left(A_{i t}, d_{i t}=d, R_{i t}, X_{i t}, \mu, \epsilon_{i t}\right)=\bar{U}_{i t}^{h}\left(A_{i t}, d_{i t}=d, R_{i t}, X_{i t}, \mu\right)+\epsilon_{i t}^{d h} .
$$

In the empirical implementation below, $\epsilon_{i t}^{d h}$ is simply an additive econometric error; however, in the theoretical model, $\epsilon_{i t}^{d h}$ is given a structural interpretation as an unobserved state variable (Aguirregabiria and Mira, 2010). The alternative specific lifetime value function in health state $h$, conditional on unobserved heterogeneity $\mu$, is:

$V_{d}^{h}\left(S_{i t}, \epsilon_{i t}^{d h} \mid \mu\right)=\bar{U}_{i t}^{h}\left(A_{i t}, d_{i t}=d, R_{i t}, X_{i t}, \mu\right)+\epsilon_{i t}^{d h}+\beta\left[\left(1-\varsigma_{i t+1}\right) \sum_{a=0}^{1} \pi_{i t+1}^{a} E_{i t}\left[V^{a}\left(S_{i t+1} \mid \mu\right) \mid d_{i t}=d\right]\right]$.

Here, $V^{a}\left(S_{t+1} \mid \mu\right)$ is the maximal expected lifetime utility of being in health state $a$ in period $t+1$. The value function is conditional on the unobserved heterogeneity component $\mu$. The expectation operator is taken over the time $t$ posterior distribution of $\theta$, as well as other shocks that determine future state variables and preference shocks. Given the unitary dimension of the posterior distribution, as well as the i.i.d. nature of other shocks to the model, I use a Monte Carlo method to evaluate the expectation within solution to the model $\cdot^{19}$ Let $\bar{V}_{d}^{h}(\cdot)=$ $V_{d}^{h}(\cdot)-\epsilon_{i t}^{d h}$. If we assume that $\epsilon_{t}^{d}$ has an Extreme Value Type I distribution, then the maximal (EMAX function) expected lifetime utility has the following closed form solution:

$$
V^{h}\left(S_{i t+1} \mid \mu\right)=E C+\ln \left(\sum_{d=1}^{D} \exp \left(\bar{V}_{d}^{h}\left(S_{i t+1} \mid \mu\right)\right)\right) \quad \forall t, \quad \forall h .
$$

Here, EC is Euler's constant. Furthermore, because the error term $\epsilon_{t}^{d}$ is additively separable, the conditional choice probabilities take the following dynamic multinomial logit form:

$$
p\left(d_{i t}=d \mid S_{i t}, \mu\right)=\frac{\exp \left(\bar{V}_{d}^{h}\left(S_{i t} \mid \mu\right)\right)}{\sum_{d=0}^{2} \exp \left(\bar{V}_{d}^{h}\left(S_{i t} \mid \mu\right)\right)} \forall t, \forall h
$$

To preview the empirical implementation, the conditional smoking choice probability in equation 11 enters the likelihood function. The parameters that dictate the choice probability are structural in the sense that they are follow from the above maximization problem. Also to enter the likelihood function are the health and death transition probabilities, as well as health marker index and smoking stock transition equations.

\footnotetext{
${ }^{19}$ See Appendix A for additional details.
} 


\section{Data: The Framingham Heart Study}

The Framingham Heart Study is one of the longest running panel studies in the world. With the stated goal to "identify the common factors that contribute to cardiovascular disease", the study contains repeated observations of individuals over a 50 year period. ${ }^{20}$ Beginning in 1948, the Framingham Heart Study began collecting biennial health data from 5107 individuals living in Framingham, Massachusetts. These individuals formed what became known as the Original Cohort. In 1971, the Framingham Heart Study began following the offspring of the Original Cohort to form the Offspring Cohort. Each cohort represents a different panel study that has continued into the 21st century. The main drawback of these data is that all participants in the study are from Framingham. Therefore, there is no geographic, and limited demographic, variation. Another drawback of these data is the lack of income measures. However, the data contain a wealth of health and smoking information that are ideal for analyzing the trade-off between smoking and the potential for future health shocks.

The structural model above is estimated with data from the Framingham Offspring Cohort. ${ }^{21}$ The decision to focus on data from the Offspring Cohort stems from the consistency with which the health exams were administered. Smoking and health questions changed over time in the Original Cohort; thus, constructing uniform measures of smoking history, perperiod behavior, and health variables (especially health markers) proved to be difficult. In constructing the sample used in estimation, I drop all individuals with a missing exam and all those lost to attrition. ${ }^{22}$ Table 1 explains my process of sample construction. ${ }^{23}$ The final sample consists of 19,461 person/year observations.

\section{III.1 Sample Statistics}

The sample statistics given in this section are by Framingham Heart Study exam. Offspring Cohort health exams have been administered at roughly four year intervals from 1971

\footnotetext{
${ }^{20}$ The Framingham Heart Study: http://www.framinghamheartstudy.org/index.html

${ }^{21}$ In another study, I am examining the intergenerational transfer of smoking preferences between Original and Offspring Cohort participants.

${ }^{22} 671$ individuals are lost to attrition (i.e., some reason other than death) at some point during the seven exams. This constitutes approximately $18 \%$ of my sample. The decision to drop these individuals is based on the computational tractability of modeling attrition. Simple t-tests for difference of means suggest that those that attrit are slightly more likely to be women, have a three point lower level of systolic blood pressure on average, and have a statistically insignificant difference in coronary heart disease incidence than their nonattriting counterparts. Those that attrit are on average slightly more likely to smoke.

${ }^{23}$ The full sample contains 5124 individuals. For this work, I only have access to data for those individuals from whom consent for distribution was granted.
} 
to the present. While variation in the timing of the health exams may seem detrimental to implementing the structural model above, as I discuss in Section IV, in estimation I exploit this variation to help identify the model. Indeed, Section IV provides the majority of the sample statistics for data used in estimation. In the empirical implementation of the model, I expand these data to reflect the yearly decision making model presented above. I therefore postpone the presentation of smoking and health transition statistics by age until the empirical implementation description in section IV.

I have data for each individual in the Framingham Heart Study Offspring Cohort for up to seven health exams. Individuals range in age from 13 to 62 at the first exam which was conducted between 1971 and 1975. For each participant, subsequent exams occurred at varying time intervals. Table 2 provides information on the average timing of each exam across individuals, in addition to demographic information. Because attrition has been eliminated, the number of individuals at each exam reflects only those that have survived. Over the health exams, the sample becomes slightly more weighted toward female and non-married individuals. For confidentiality reasons, all study participants are white. Table 2 also shows the great variability in ages across the sample. At the first exam, there are individuals who are as old as the average age at the final exam. Indeed, over the entire sample, ages range from 13 to 88. Table 3 gives sample percentages of the maximum number of years of education by category. The sample reflects a rather well educated cohort for the time period. Nearly $89 \%$ of the sample has a high school degree or better.

Table 4 breaks down the sample by smoking prevalence over exams. Over the seven exams, the sample smoking prevalence drops from roughly $41 \%$ to $11 \%$. Interestingly, at the first exam, smoking prevelence in the sample is roughly consistent with that of the United States average prevelence (37\% of Americans smoked in 1973). However, by the final exam, the sample percentage of smokers has decreased to roughly $11 \%$ whereas the national average fell to only $23.3 \%$. The sample is also clearly older than the general population by the seventh exam. $^{24}$

Table 5 shows the percentage of the sample living with a chronic condition at each health exams. I define a chronic condition to include a wide variety of cardiovascular diseases (e.g., coronary heart disease, myocardial infraction, cerebrovascular accidents, congestive heart failures, etc.) and cancers (lung, larynx, tongue,esophagus, etc.). ${ }^{25}$ The decision to aggregate the

\footnotetext{
${ }^{24}$ Centers for Disease Control and Prevention: http://www.cdc.gov/tobacco/basic_information/index.htm

${ }^{25}$ Due to data limitations, the dichotomous variable for chronic health used throughout does not capture all diseases that are caused by smoking. For example, the Framingham Heart Study data do not include panel data for chronic obstructive pulmonary disease (COPD). Given that COPD is the number four leading cause of death in the United States ${ }^{26}$, the omission of COPD in the chronic health indicator may understate the importance of both disease in the probability of death and the extent to which preferences for smoking vary across chronic health states.
} 
data to this level stems from the computation burdens of estimating additional parameters in the structural model. As in the theoretical model, I assume that upon transiting to a chronic health state, an individual remains in that state for life. The incidence of new chronic conditions is in column 3 of Table 5 .

\section{Empirical Implementation}

In estimating the model described in Section II with the data described in Section III, there are four hurdles. The first main hurdle lies in the timing of the health exams. While the data contain only seven exams over a 40-year period, the theoretical model is based on a yearly decision making process over a finite time horizon. As explained below, I exploit retrospective questions in the data to construct a dataset that mirrors the timing of the model. This process generates yearly data for all variables in the theoretical model except health marker data for years in which no health exam took place. To overcome this problem, I use predictions from solution to the model to integrate over "off years" as well as to explain the initial condition for each individual. Given the expanded dataset, the second hurdle is that state variables $A_{i t}$ and $R_{i t}$ must be constructed from the Framingham Heart Study data in such a way as to capture an individual's smoking history and health markers, respectively. For each of these variables, I employ principal component analysis in a method similar to that of Sickles and Williams (2008). The third hurdle is the identification of the model parameters. As I describe below, variation in the timing of health exams across individuals helps to identify the model. Finally, the last hurdle involves modeling permanent unobserved heterogeneity. This section ends with a discussion of my solution method, likelihood function construction, and maximization routine.

\section{IV.1 Health Exam Timing}

While I observe individuals at only seven health exams over a 40-year period, the theoretical model is based on a yearly decision making process. To reconcile this difference, I proceed in the following steps. First, in solution to the model, I specify the final period, $T$, to be at age 100. That is, the probability of death at the end of period $T$ equals one. The yearly model is then solved recursively back to age 7 , at which point I assume that all individuals have a smoking stock of zero (i.e. $A_{i 7}=0, \forall i$ ). Second, the data from Section III are expanded based upon retrospective questions. With the exception of the health marker information needed to construct the health marker index, $R_{i t}$, data are available to construct a yearly dataset from age seven until an individual either dies or completes his or her seventh exam. Data in years prior to an individual's first exam were constructed based on questions at 
the first and second exams that asked, if applicable, the first age at which one started smoking and the age at which one stopped smoking. For later years in between health exam years, smoking data were imputed based on history and adjacent health exam data. Specific dates are available in the data for any chronic health and mortality events.

Figure 1 shows the sample probabilities for each smoking choice by age. Because figure 1 reports smoking percentages by age, for older ages, the data become noisy because of either right-censoring or death. Of the 3008 observations considered, 15\%, or 464 individuals, leave the sample through death. Table 6 shows general smoking summary statistics from the expanded data. On average, if an individual ever smokes, he/she starts just before age 20, although the median age is 18 . Table 7 reports smoking behavior transitions around health exams and chronic health shocks, as well as the overall average transitions. Conditional upon an individual's smoking behavior one period prior to each event, the table reports percentages in each smoking option one and three years after the event. For example, of those individuals smoking heavily one period prior to a chronic health shock, $70.24 \%$ continued to smoke heavily one period after the shock and $47.06 \%$ were still smoking heavily three periods after the shock. In both event cases, more individuals had quit smoking three years post as compared to one year post, but considerably more had quit three years after an event than the baseline set of transitions. The table provides at least antidotal evidence that each event (health exams and chronic health shocks) alters smoking behavior in the sense that the magnitudes of the transitions, both one and three years post, are larger than the overall transitions.

\section{IV.2 Continuous State Variable Construction}

For the model to both remain computationally tractable and be consistent with the assumption of conjugate distributions, I need continuous, scalar representations of both an individual's smoking history and her health markers. For both the health marker index and the smoking stock, I use the first principal component of a principal component analysis (PCA) procedure to capture these variables. The first principal component is constructed as a linear combination of data and of factor loadings from the highest eigenvalue eigenvector from an eigenvector decomposition of the variables' correlation matrix. The trade-off with PCA is both completeness and interpretation. Only considering the first principal component implies that any remaining variation in the data (i.e. the second, third, fourth, etc. principal components) is lost. Furthermore, because the weights used to construct the index are derived only from the correlation between the variables themselves, the relative magnitudes of the weights may come into question when predicting an outcome of interest (e.g., mortality). In the context of most structural models, however, reducing the dimension of the data is clearly advantageous. 
In constructing $R_{i t}$, the health marker index, I use PCA with the following (standardized) variables: systolic blood pressure, diastolic blood pressure, total cholesterol, high-density lipoprotein (HDL) cholesterol, and a diabetes dummy. PCA is most effective when there exists significant correlation between the variables. As one might expect, the correlation between these health markers is high. As noted in Section III, these health markers are identified by the Framingham Heart Study as significant predictors for an individual's general 10-year risk of cardiovascular disease. ${ }^{27}$ The first principal component of these variables explains approximately $33 \%$ of the total variation. Unfortunately, this implies that two-thirds of the variation in health markers is being lost. However, I now have a continuous index of health markers. I see two main justifications for using the first principal component as my measure for the health markers. First, the theory places no restriction on the amount of information that $R_{i t}$ must convey, only that it conveys some information. Any computationally tractable definition of $R_{i t}$ will have to be an approximation. That I can explain a third of the variation in the variables that the medical literature view as signficant will at least inform to some degree. Second, most papers that use PCA use first principal components that explain between $20 \%-40 \%$ of the total variation. ${ }^{2829}$

To provide intuition as to the weights used to create the health index, Table 8 presents the eigenvector values associated with each health marker. In this context, the continuous health index can be interpreted as a measure of bad health (i.e. higher values of the index imply worse overall health). Note in Table 8 that only HDL, or "good" cholesterol, negatively affects the health index.

Turning to the smoking stock, as discussed above, the smoking stock summarizes all past smoking decisions prior to period $t$. Again using PCA, I define the index $A_{i t}$ as the first principal component of the following four standardized variables: total number of years smoking at time $t$ (experience), number of years smoking at time $t$ since last year not smoking (tenure), number of years at time $t$ not smoking since last year smoking (cessation), and the intensity

\footnotetext{
${ }^{27}$ While influential in predicting cardiovascular disease, there is no evidence that suggests that these health markers predict different forms of cancer. In the context of the model, a summary of these health markers will have less predictive power on the chronic health state if that state is defined as an aggregation of cardiovascular disease and cancer. However, these markers still provide an overall assessment of an individual's health. The extent to which these markers may influence smoking behavior through the Bayesian updating process is an empirical question.

${ }^{28}$ In the context of socioeconomic indices, see Vyas and Kumaranayake (2006) for a good overview of PCA.

${ }^{29}$ As an alternative to using the PCA to construct the health marker index, I have also considered using the weights suggested by the Framingham Heart Study General Cardiovascular Disease, 10-year risk assessment. The weighted index of health markers from Framingham is highly correlated with the PCA index used in this paper (correlation coefficient of 0.79). Furthermore, in reduced-form regressions, the PCA generated index better predicts chronic health and mortality outcomes.
} 
of smoking in the previous period, $t-1 .{ }^{30}$ The first principal component explains nearly $52 \%$ of the total variation in these four variables.

To aid in interpretation of both the resulting smoking stock and the associated parameters to be estimated, I normalize the smoking stock as follows. First, I run PCA on just those with some smoking history. That is, individuals with any observed or reported past smoking in each period are included in the PCA. For example, if an individual takes her first exam at age 18 and begins smoking at age 22, all observations from this individual after age 22 are included in the PCA, whereas observations prior to 22 are not included. Second, I shift the distribution of the resulting index such that the person with the lowest value has a stock approximately equal to zero. Finally, for individuals with no smoking history, I assign a stock value of zero. Table 9 reports the weights on the four variables of interest in the smoking stock. Notice that while experience, duration, and smoking intensity of an individual all increase the stock index, cessation from smoking decreases the stock. Therefore, I interpret higher values of the index as more accumulated smoking stock capital.

\section{IV.3 Identification}

Generally, the structural model is identified from the variation in the timing of the health exams. While the number of years between health exams does not directly affect health ${ }^{31}$, observationally equivalent individuals with different time gaps between exams may select different smoking patterns. The different smoking patterns may arise because different time gaps will induce variation in the belief distribution across individuals. For example, if, after completing their second health exam at the same time, one individual receives her next exam in three years while another individual receives her next exam in five years, the data may show different smoking patterns during the two years in which the first individual had a different set of beliefs. Indeed, the transition matrix in Table 7 shows that individuals change their smoking behavior around health exams more than average. Table 10 provides a sense of the variation in the number of years between exams (the vertical tab) for each exam (the horizontal tab). While the gap between the first and second exam is clearly the longest, and the time gap shrinks at later exams, each exam exhibits considerable variation across individuals in the number of years to be administered.

\footnotetext{
${ }^{30}$ Intensity is measured as the average number of cigarettes per day. Each of these smoking variables is measured as the value entering the examination.

${ }^{31}$ The considerable time lag between exams is because the Framingham Heart Study administers health exams in time "windows". However, there is no evidence to suggest that those with worse health markers select into smaller time gaps between exams. Indeed the correlation coefficient between the health marker index and the number of years between exams is -0.008
} 
The following set of parameters are to be estimated.

Utility Parameters:

Health Transition Parameters:

Death Transition Parameters:

Smoking Stock Parameters:

Learning and Risk Parameters:

Factor Loadings:

$$
\begin{aligned}
& \Theta_{U}=\left\{\alpha_{0 h}, \ldots, \alpha_{11 h}\right\}_{h=0}^{1} \\
& \Theta_{H}=\left\{\lambda_{0}, \ldots, \lambda_{10}\right\} \\
& \Theta_{M}=\left\{\omega_{0}, \ldots, \omega_{12}\right\} \\
& \Theta_{A}=\left\{\delta_{1}, \delta_{2}, \delta_{3}, \sigma_{\eta}\right\} \\
& \Theta_{R}=\left\{\bar{\theta}, \sigma_{\theta}, \sigma_{v}, \phi, \zeta\right\} \\
& \Theta_{\rho}=\left\{\left\{\left\{\rho^{U h d}\right\}_{h=0}^{1}\right\}_{d=0}^{2}, \rho^{H}, \rho^{M}, \rho^{R}, \rho^{A}\right\}
\end{aligned}
$$

Additionally, I estimate the probability weights of the mass points for the discretized distribution of the permanent unobserved heterogeneity, $\mu$. Let $\Theta=\left\{\Theta_{U}, \Theta_{H}, \Theta_{M}, \Theta_{A}, \Theta_{R}, \Theta_{\rho}\right\}$. In order to identify the preference parameters, I normalize the utility of death to be zero. Relative to this normalization, identification of the preference parameters comes mainly from variation in smoking behavior and health and death transitions over time. For example, different smoking choices across the smoking stock, health marker index, and age levels identifies the interaction preference parameters. Furthermore, the withdrawal parameter, $\alpha_{9}$, is identified off of variation in the choices of individuals after a period in which an individual quits. Thus, conditional upon having smoked in the previous period, both the reinforcement, $\alpha_{2}$. and $\alpha_{6 \text {. }}$, and withdrawal effects, $\alpha_{9}$, encourage current period smoking. However, withdrawal is separately identified from the reinforcement effects because, while the smoking stock variable depreciates at rate $\delta_{1}$ following cessation, the utility cost paid from withdrawal only lasts one period. Finally, the direct impacts of the stock on utility, $\alpha_{10}$. and $\alpha_{11}$, reflect tolerance in smoking and are identified by individuals that progress from light to heavy smoking.

In the absence of subjective expectation data, the structure of the model is needed to identify the presence of learning. Mira (2007) notes that learning can no more be identified than can rational behavior in the sense that, the model assumes that behavior (learning) follows from the defined structure. If, however, the prior distribution of beliefs is proved to be degenerate (i.e., if the null hypothesis that $\sigma_{\theta}=0$ is not rejected), then the results would suggest an absence of learning. The identification strategy of the specific learning parameters is therefore quite subtle. While identification of $\bar{\theta}$ comes from variation in the smoking stock and health marker index, variation in smoking by individuals over time identifies $\sigma_{\theta}$ (Crawford and Shum, 2005). If, indeed, individuals are learning over time, choices at the end of the time frame relative to the beginning should better reflect an individual's true match value, $\theta_{i}$. An additional source of variation that helps to identify the learning parameters is the variation across individuals in the timing of health exams. There exists considerable variation in the number of years between exams across individuals; thus, two similar individuals that receive health information at different frequencies may develop different smoking patterns. Because of the assumption of conjugate normal distributions, identifying the mean and variance of 
$\theta_{i}$, in addition to the variance of $v$, which is identified from the health marker index transition equation, is sufficient to characterize the learning process. Finally, the identification of chronic health and death transition parameters comes from variation in the state variables and the observed incidence of chronic health and death.

\section{IV.4 Permanent Unobserved Heterogeneity and Initial Conditions}

Permanent unobserved heterogeneity enters the model in a linear fashion through the $\mu$ term and the associated factor loadings. The factor loadings allow for a different effect of the unobserved $\mu$ term everywhere it enters. Rather than placing a distributional assumption on the underlying unobserved heterogeneity, I approximate its distribution with a step function and estimate the factor loadings and mass point probabilities with other parameters in the model (Heckman and Singer, 1984). This discrete factor method has been shown to approximate both Gaussian and non-Gaussian distributions well (Mroz, 1999).

I first observe individuals at various points in their life cycle (i.e., different ages at the first health exam) and with a variety of health histories. Failing to properly model these histories would lead to an initial conditions problem. Furthermore, the initial conditions problem may lead to an issue of dynamic selection into smoking behaviors. That is, individuals in some permanently lower (unobserved) health state may select into smoking. However, solution to the model generates individual probabilities of choice behavior and health/death transitions for all ages beginning at age seven. Recall that data exist for all smoking, chronic health, and death events from age seven until either death or the final health exam (exam 7 in the data). At age seven, I assume that each individual has a smoking stock of zero and has no chronic health problems. The only remaining initial condition is the initial health marker index upon entering the sample. Using the model, I can simulate a health marker index for each period from age seven until the first observed health exam. Hence, I use the model to generate probabilities of an individual's health history when they are first observed in the sample (Khwaja, 2010). Individuals enter the sample aged between 13 and 62 years. At age seven, I assume that the lagged value of the health marker index is in the 90th percentile (e.g., good health) of each health marker that is used to construct the index. I then use the weights from the principle component analysis to construct the lagged value. Recall that the simulated health marker index is scaled by demographic characteristics, $X_{i t}$, as well as the unobserved heterogeneity, $\mu$, term and its factor loading. Furthermore, individual variation in the data at the first exam (the initial condition) helps to identify parameters of the model. 


\section{IV.5 Likelihood Function}

Solution of the model yields the conditional choice probabilities that enter the likelihood function. In solving the model, I use a variant of the Keane and Wolpin (1994) value function interpolation method for approximating the value function. Appendix A thoroughly explains my solution technique.

The parameters of the model are estimated via simulated maximum likelihood. In constructing the likelihood function, consider first the contribution of individual $i$. Given that $\eta_{i t} \sim \mathscr{N}\left(0, \sigma_{\eta}^{2}\right)$ and $v_{i t} \sim \mathscr{N}\left(0, \sigma_{v}^{2}\right)$, I can express the probability density functions of $A_{i t}$ and $R_{i t}$ respectively as: ${ }^{32}$

$$
\Lambda_{t}=f\left(\eta_{i t} \mid A_{i t-1}, d_{i t-1}, \mu, \rho_{A}, \Theta_{A}\right)=\frac{1}{\sigma_{\eta}} \phi\left(\left[\log A_{i t}-\delta_{1} \log A_{i t-1}-\delta_{2} d_{t-1}-\rho_{A} \mu\right] / \sigma_{\eta}\right)
$$

and

$$
\Omega_{t}=g\left(v_{i t} \mid X_{i t}, \kappa_{i t}, \mu, \rho_{R}, \Theta_{R}\right)=\frac{1}{\sigma_{v}} \phi\left(\left[R_{i t}-\zeta R_{i t-1}-X_{i t} \phi-\kappa_{i t}-\rho^{R} \mu\right] / \sigma_{v}\right)
$$

where $\phi(\cdot)$ is the standard normal distribution. Recall, however, that the health marker index, $R_{i t}$, and only the health marker index, is unobserved in periods in which a health exam was not taken. ${ }^{33}$ I must, therefore, integrate over $R_{i t}$ in all periods with no health exam. For ease of exposition, define the dummy $y$ as follows:

$$
y_{i t}=\left\{\begin{array}{lll}
1 & \text { if } & \text { An exam was taken in year } \mathrm{t} \\
0 & \text { if } & \text { No exam was taken in } \mathrm{t}
\end{array}\right\}
$$

Let $w$ index the draw from the Monte Carlo simulator. Define $Z_{i t w}^{y=1} \mid \mu$ as individual $i^{\prime} s$ likelihood contribution in period $t$ for draw $w$ when $y_{i t}=1$ and conditional on unobserved heterogeneity term $\mu$ :

$$
Z_{i t w}^{y=1} \mid \mu=\prod_{d=0}^{2}\left(p\left(d_{i t}=d \mid s_{i t}, \mu\right) * \Lambda_{t} * \Omega_{t} * \prod_{h=0}^{1}\left(\pi_{t+1}^{d h} \mid \mu\right)^{\mathbf{1}\left[H_{i t+1}=h\right]} * \prod_{m=0}^{1}\left(\varsigma_{t+1}^{d m} \mid \mu\right)^{\mathbf{1}\left[M_{i t+1}=m\right]}\right)^{\mathbf{1}\left[d_{i t}=d\right]} .
$$

Here, $\pi_{t+1}^{h w}$ represents the probability of transiting to health state $h$ in period $t+1$ and $\varsigma_{t+1}^{m w}$ is the probability of transiting to death state $m$ in period $t+1$, given draw $w$. Unless a health exam was taken in the period directly before $t$, the lagged value of the health marker index in equation 13 is unobserved. In practice, I use the expected health marker index given the

\footnotetext{
${ }^{32}$ Note that the $i$ subscript has been dropped from the permanent unobserved component, $\mu$. As is shown below, in the empirical model, the distribution of $\mu$ has been discretized to $K$ points of support and is integrated out of the likelihood function.

${ }^{33}$ All right-hand side terms in the health marker equation are observed in these "off" years due to retrospective questions and/or imputation with the exception of the lagged value of the health marker index when the previous period did not contain a health exam.
} 
model parameters and the lagged value. In periods in which $y_{i t}=0$, define the expected health marker index, conditional on the model parameters as:

$$
\widetilde{R}_{i t}=\mathrm{E}_{v}\left(R_{i t} \mid \Theta_{R}, S_{i t}, \mu\right)
$$

Here, the expectation operator is taken over the i.i.d. noise term, $v$. Other probabilities in the model are conditional on $\widetilde{R}_{i t}$ for years in which $y_{i t}=0 .{ }^{34}$ In the period directly after a health exam, the lagged value of the health marker index (i.e., from the exam and not the simulated term) is used in the construction of $\widetilde{R}_{i t}$. Therefore, define $Z_{i t w}^{y=0} \mid \mu$ as individual $i^{\prime} s$ likelihood contribution in period $t$ when $y_{i t}=0$ :

$$
\prod_{d=0}^{2}\left(p\left(d_{i t}=d \mid s_{i t}, \widetilde{R}_{i t}, \mu\right) * \Lambda_{t \mid \widetilde{R}_{i t}} * \prod_{h=0}^{1}\left(\pi_{t+1}^{d h} \mid \widetilde{R}_{i t}, \mu\right)^{1\left[H_{i t+1}=h\right]} * \prod_{m=0}^{1}\left(\varsigma_{t+1}^{d m} \mid \widetilde{R}_{i t}, \mu\right)^{1\left[M_{i t+1}=m\right]}\right)^{1\left[d_{i t}=d\right]} .
$$

The total conditional (on $\mu$ ) likelihood contribution from individual $i$ for all time periods $7, \ldots, T_{i}$, where $T_{i}$ is either the period of an individual's death or their final exam, is:

$$
L_{i w}(\Theta \mid \mu)=\prod_{t=7}^{T_{i}}\left[\prod_{y=0}^{1}\left(Z_{i t w}^{y} \mid \mu\right)^{1\left[Y_{i t}=y\right]}\right] .
$$

Because of the discretized distribution of the unobserved heterogeneity, each individual's unconditional contribution will be a finite mixture of likelihoods. Given $K$ points of support in the estimated distribution of $\mu$, the unconditional likelihood function contribution for individual $i$ is:

$$
L_{i w}(\Theta)=\sum_{k=1}^{K} \xi_{k} L_{i w}\left(\Theta \mid \mu_{k}\right)
$$

Where $\xi_{k}$ is the estimated probability weight placed on mass point $k$. Individual $i$ 's contribution to the likelihood is then the average contribution over the $w$ draws. The full sample log-likelihood function is:

$$
L(\Theta)=\left[\sum_{i=1}^{N} \log L_{i}(\Theta)\right] .
$$

The parameter estimates in $\Theta$ are estimated via a nested solution method (Rust, 1987). The inner algorithm solves the dynamic model for each individual conditional on a given set of parameters and for all mass points of the unobserved heterogeneity distribution. ${ }^{35}$ Using the resulting probabilities, the outer algorithm calculates the unconditional likelihood function, $L(\Theta)$, and attempts to improve the likelihood value via a BHHH gradient method.

\footnotetext{
${ }^{34}$ For years in which there was no health exam, the draw $w$ also includes a draw of the contemporaneous $v$ such that I may integrate over $R$.

${ }^{35}$ See Appendix A for a discussion of the inner algorithm.
} 


\section{Results}

Table 11 reports the main parameter estimates and their corresponding asymptotic standard errors. The estimated utility constants, $\alpha_{00}$ and $\alpha_{01}$, for the absence of a chronic health condition and a chronic health condition respectively, are quite intuitive given that the utility of death has been normalized to zero. The total marginal utility of current period light and heavy smoking is a function of $\alpha_{1} \ldots \alpha_{8 .}$. A key component of rational addiction theory, indeed the defining feature of an addictive good under rational addiction, is that past consumption reinforces current consumption. That is, the marginal utility of smoking is increasing in the smoking stock. My results are consistent with this adjacent complementarity defined in Becker and Murphy (1988). In the absence of a chronic illness, both light and heavy smoking are found to be reinforcing (i.e., $\alpha_{20}, \alpha_{60}>0$ ). Indeed, I find that heavy smoking is much more "reinforcing" than light smoking. My results also suggest that the marginal utility of light smoking in the absence of a chronic condition is invariant to the health marker index but increasing in age. Interestingly, the marginal utility of heavy smoking is decreasing in the health marker index and invariant to age when free of a chronic condition; however, when chronically ill, the marginal utility of heavy smoking is increasing in the health markers $\left(\alpha_{71}=0.001\right)$ and decreasing in age. Withdrawal from smoking, (i.e., smoking in period $t-1$ and not smoking in period $t$ ) is negative for all health states and larger in magnitude when free of a chronic illness. The withdrawal effect, in addition to the strong reinforcement effect, both drive smokers to continue smoking. Finally, the tolerance effect $\left(\alpha_{10}\right)$ flips sign across health states. In the absence of a chronic condition, smoking is found to have a tolerating effect (i.e., lower utility from a larger smoking history).

Several interesting trends emerge from these results. First, note that baseline marginal utility of both light and heavy consumption is negative with the exception of heavy smoking with a chronic condition. As suggested by the rational addiction literature, the model cannot explain why individuals start smoking. Consider that over $90 \%$ of smokers in the data start smoking before age 25 and no individuals in the data under the age of 25 have a chronic condition. The estimated preference parameters in the absence of a chronic illness suggest that, for a never smoker under the age of 25 , there is no incentive to begin smoking because the marginal utility of smoking is negative. Furthermore, the dynamic considerations of the model suggest that smoking will increase the probability of future chronic illness and death through the smoking stock and the health marker index. However, upon commencing smoking, the

resulting positive smoking stock drives the dynamics forward. Competing effects for a new smoker include the reinforcement and withdrawal effects, which both encourage more smoking, and the increased probability of chronic disease and death, which encourage cessation. 
The second main trend from the estimated preference parameters is the reversal in sign of several preference parameters upon succumbing to a chronic illness. The baseline marginal utility of heavy smoking when in the chronic health state $\left(\alpha_{51}\right)$ flips to positive. Along with the positive reinforcement $\left(\alpha_{21}, \alpha_{61}>0\right)$ and the flip in the sign of the effect of the stock on utility $\left(\alpha_{101}>0\right)$, individuals now face a positive marginal utility from heavy smoking.

The model finds evidence of a wide degree of individual variation in the effect of the smoking stock $\left(A_{i t}\right)$ on the health marker index $\left(R_{i t}\right)$ as the estimated standard deviation of $\theta, \sigma_{\theta}$, is large relative to its mean $(\bar{\theta})$. Recall further that the null hypothesis of $\sigma_{\theta}$ equaling zero is my explicit test for the presence of learning. While the results do suggest the presence of learning, the signals received at each health exam are quite noisy. The estimated standard deviation of the random error term $\left(\sigma_{v}\right)$ is large relative to $\bar{\theta}$ and $\sigma_{\theta}$.

Table 12 provides estimates of all other estimated model parameters. These estimates are not marginal effects and therefore are difficult to interpret because each outcome (health marker index, chronic health, death, etc.) is a complex function of entering period states and per-period decisions. In the simulation subsection below, I describe the results of simulations that isolate the effects of each variable on the system. However, a casual interpretation of the results in Table 12 does yield some interesting insights. The parameter estimates of the smoking stock evolution equation indicate that an individual's stock of smoking depreciates faster than suggested by the medical literature. $\delta_{1}$ suggests that, given cessation from smoking over the cycle of one year, the smoking stock is reduced by approximately $57 \%{ }^{36}$ In the context of the model, 57\% depreciation implies that after about six years of smoking cessation, an individual may have roughly the same health marker index and chronic health and death tranistion probabilities as a lifelong nonsmoker, all else equal. Additionally, the estimated magnitude of investment return in the smoking stock is greater for heavy compared to light smoking $\left(\delta_{2}<\delta_{3}\right)$.

As noted above, the estimated mean effect of the smoking stock on the health markers is positive $(\bar{\theta}=0.003)$. A greater smoking history therefore implies a higher, and thus worse health marker index. According to Table 12, a higher health marker index implies a higher probability of chronic illness (through the positive sign on $\lambda_{1}$ ), albeit at a decreasing rate $\left(\lambda_{2}<0\right)$, and death (through the positive signs on $\omega_{1}, \omega_{2}, \omega_{3}, \omega_{5}$, and $\omega_{6}$. Furthermore, given a chronic illness, the probability of death is lower during the 1980s $\left(\omega_{7}<0\right)$ and 1990s $\left(\omega_{8}<0\right)$ both relative to before 1980 to capture exogenous advances in medical technology over time.

\footnotetext{
${ }^{36}$ Note that while this suggests a large amount of depreciation, the factor loading on unobserved heterogeneity for the stock equation slows that depreciation.
} 
The model is estimated with three points of support for the discretized unobserved heterogeneity distribution. Heterogeneity located to the right of the distribution is associated with a greater likelihood of experiencing both chronic health and mortality shocks. Furthermore, the health and smoking alternative specific factor loadings in the utility function suggest that, when free of a chronic illness, the marginal utility of smoking is shifted upward for individuals with higher values of the unobserved heterogeneity. These effects are exacerbated by the fact that this type of heterogeneity also implies a smoking stock that depreciates less rapidly and never fully depreciates. Even worse, this heterogeneity characterizes continued future smoking (through the reinforcement and withdrawal effects) which also effects chronic health and death shocks (through the positive signs on $\lambda_{5}$ and $\omega_{4}-\omega_{6}$ ). The positive factor loadings on the marginal utility of smoking, along with the positive factor loading on mortality, suggest a strong positive correlation between the underlying factors that influence both outcomes. Indeed, these results suggest that individuals that are more likely to smoke, are also more likely to die independently of smoking. I provide further evidence of this correlation in the model simulation section below.

\section{V.1 Model Fit}

Figure 2 summarizes the relationship between the model's predicted probabilities and the observed data by age. Each pane of the figure represents one specific smoking option. For each individual, I compare observed smoking decisions and predicted smoking probabilities for periods up to either her final exam (exam seven) or death. I then average the results across individuals at each age. ${ }^{37}$ The model predictions generated from the solution routine fit the data well even at ages for which there are not many observations.

Table 13 reports sample and predicted smoking probabilities by health exam and health state. I do not include a table on model fit by exam conditional on being in the chronic health state because less than one, three, and seven percent of individuals have a chronic condition in exams one, two, and three respectively. Note however that the average predicted choice probability across all health exams conditional on being in the chronic health state mirrors the observed probabilities in the data fairly well. Table 13 suggests that the model does a good job of predicting whether or not an individual smokes at all. The model slightly under predicts light smoking and slightly over predicts heavy smoking.

Figure 3 compares the observed sample probabilities of chronic health with the predicted health probabilities, as generated by the model at the estimated parameter values. As in Figure 2, Figure 3 averages predicted and sample probabilities across individuals by age only for those

\footnotetext{
${ }^{37}$ Despite the fact that the model is solved from age 7 to 100 , the figure only presents results for ages 20 to 75 . Outside of the 20 to 75 age range, there are insufficient data for an informative comparison.
} 
individuals with an observation at that age. Figure 3 reflects both transitions to and surviving members of the chronic health state. This is because solution to the model yields a predicted probability of transiting to a chronic health state of one for individuals already in that state. Note that for most ages, the model slightly over predicts the probability of being in a chronic health state. 


\section{V.2 Model Simulation}

In this section, I simulate smoking behavior and health outcomes using the structural model and the estimated parameters. I address how smoking affects morbidity and mortality outcomes as well as how learning from personalized information may impact these behaviors and outcomes. My simulations proceed as follows. First, I construct a simulated sample of 1000 individuals that mirrors the joint distribution of observable demographic characteristics (education, gender, marriage, and initial age upon entering the Framingham Study) of the Framingham sample. Next, for each simulated individual $i$, I construct 50 sets of match value, unobserved heterogeneity, and error draws over the estimated time frame.

$$
\left\{\theta_{i k}, \mu_{i k},\left\{v_{i k t}, \eta_{i k t},\left\{\epsilon_{i k t d}\right\}_{d=0}^{2}\right\}_{t=7}^{100}\right\}_{k=1}^{50}
$$

Smoking behavior and health outcomes are then simulated for each of the 50,000 observations from age seven until death.

First, I reconstruct Table 7 using the simulated smoking behavior to evaluate the model's performance in capturing smoking transitions around significant events. These results are reported in Table $14 .{ }^{38}$ For those simulated to be not smoking prior to a health exam or a chronic health shock, the simulated smoking probabilities one and three periods after these events mirror those from the data. The model does less well in simulating behavior conditional on lagged light or heavy smoking. While the simulated probabilities of not changing behavior after one of the two events reflect those from the data, the model tends to under predict the probability of quitting and over predict the probability of switching to a different smoking intensity. However, the model does capture the general trend that more individuals have quit three years after an event when compared to one year after.

Next, I use the simulated model to address how smoking impacts the age of chronic health onset and death. Figure 4a reports, by age, the percentage of the simulated sample with a chronic condition while forcing individuals to 1.) never smoke, 2.) smoke lightly from age 18, and 3.) smoke heavily from age $18 .^{39}$ Under these same forced behaviors, Figure 4b shows, by age, the percentage of the simulated sample that remains alive. The results in Figure 4 confirm the findings in Sloan et al. (2003) that the detrimental effects of smoking occur largely after the age of 50. Indeed, the gap in the percentage of the sample in the chronic health state between never smokers and heavy smokers widens from less than 10\% at age 50 to more than $17 \%$ at age 70 . Similarly, while the difference in those surviving to age 50 between heavy and never smokers is five percentage points, that gap widens to 30 percentage

\footnotetext{
${ }^{38}$ Transitions around health exams are unconditional on chronic illness.

${ }^{39}$ Recall from the structural model that I assume that, upon transiting to a chronic health state, an individual remains in that state for life.
} 
points at age 70. These results are roughly inline with those of Doll et al. (2004). Those authors find a difference of approximately 28 percentage points at age 70 when considering never smokers and smokers. The first half of Table 15 reports the mean age of onset for various health outcomes. Individuals who are forced to smoke lightly and smoke heavily from age 18 onwards face a mean age of chronic health onset that are approximately two and four years earlier than those forced to never smoke. While Doll et al. (2004) report that smoking shortens the lifespan by ten years, my results suggest the reduction is approximately four and eight years for light and heavy smoking, respectively. ${ }^{40}$

While Doll et al. (2004) only condition their results on decade of birth and gender, I report results that are conditional on both observed and unobserved factors. Here, I highlight the importance of incorporating unobserved heterogeneity. Figure 5 plots the same two graphs as in Figure 4 but now conditions each result by unobserved "type". Panels a. and b. report health outcomes assuming that all simulated individuals never smoke. Note that while unobserved heterogeneity does not play a significant role in chronic health transitions, the model predicts that type three individuals face lower expected longevity. Recall that the alternative specific factor loadings in the utility function greatly increase the marginal utility of smoking for individuals of a higher type. Indeed, the model predicts that only individuals with the largest mass point, type three, will ever smoke. Therefore, Figure 5 demonstrates that, independent of smoking, individuals of a higher type face lower expected longevity.

Next, I use the model to simulate chronic health and death outcomes under different lifetime smoking paths to assess the impact of smoking cessation on these outcomes. I simulate health outcomes assuming that an individual smokes heavily from age 18 and quits forever at ages 30, 40, 50, and 60. The results, reported in Table 15, imply that quitting smoking at ages $30,40,50$, and 60 years of age increases life-expectancy by approximately 8, 7.75, 7, and 5.5 years, respectively. These results suggest clear life expectancy gains from quitting at all stages of the life cycle. Figure 6 shows the survival percentages by age for the different smoking patterns. Note that for individuals that quit at age 30, their expected longevity is roughly identical to never smokers. Similarly, quitting by age 40 has minimal effects on mortality probabilities. Individuals that smoke into their fifties and sixties, however, have a much more likely chance of dying prematurely. These results, although smaller in their absolute numbers because of the treatment of the positive selection between smoking and mortality, mirror the results of Doll et al. (2004) and other studies that have examined smoking cessation.

Given the other main focus of this paper on the value of personalized health information, I next evaluate policies that alter either the timing or the frequency with which information

\footnotetext{
${ }^{40}$ Doll et al. (2004) do not take into account intensity of smoking in these calculations. My results indicate that, conditional on smoking, the intensity with which one smokes is an important factor explaining health outcomes.
} 
is received. First, to demonstrate the speed at which individuals learn, Table 16 reports the change in the average posterior variance after each health exam of the baseline simulation. Note that after the first exam (i.e., the first signal of information) the posterior variance decreases by nearly $20 \%$. By the seventh exam, the mean posterior variance has been decreased by $40 \%$. In spite of the "honing in" on individuals' true match values, smoking behavior appears to only slightly be influenced by learning.

As a natural benchmark, I compare the predictions of the baseline model to results from specifications with complete information (i.e., $\tau_{i t}=\theta_{i} \forall t$ ) and a situation where an individual undergoes yearly health exams as opposed to every two to eight years. Figure 7 presents the mean percentage difference of simulated individuals choosing each smoking option for each information scenario relative to the baseline prediction. First, note the scale of the vertical axis. Neither of these counterfactual simulations greatly alters model predicted lifetime smoking behavior. Second, any divergence in behavior from baseline appears in light smoking. Neither counterfactual scenario has any affect on the average probability of heavy smoking. Between ages 40 and 55, receiving more information about health markers (yearly exams) does slightly reduce smoking, if only by approximately $0.2 \%$. After age 55 , somewhat counter intuitively, the simulations suggest that the effect of more information, that is, yearly exams, is only to encourage individuals to smoke lightly. In the extreme, with complete information, individuals are more likely to smoke lightly at all ages.

One possible explanation for the small results of these counterfactuals is that the effect of the smoking stock on the health marker index is small $(\bar{\theta}=0.002)$ and because the estimated standard deviation of the effect is large relative to the mean. Upon learning their true match value, individuals feel that the health effects of smoking are manageable. ${ }^{41}$ Another explanation is that the health markers simply do not greatly predict chronic health and mortality outcomes. While individuals learn about their match value, they place little value on this information. Ultimately, while my results do confirm that major chronic health shocks to alter smoking behavior, I do not find evidence that information on personalized health marker information changes behavior.

\footnotetext{
${ }^{41}$ For match values that are negative, there may be an incentive to continue to smoke because an increased smoking stock will decrease the health marker index, which in turn, will lower chronic health and death probabilities. Other experiments in which health signals where amplified, that is, while the health marker index evolved according to the simulation, individuals received signals that suggested "scary" results, induced individuals to quit significantly more rapidly than the baseline results.
} 


\section{Discussion}

This study formulates and estimates a dynamic stochastic model of smoking behavior. The model extends the classic rational addiction model to allow for health learning. By estimating the structural parameters of the model, I capture preferences and expectations in the tradeoff between smoking and the potential for future health shocks. The structural approach also allows for counterfactual simulations that a.) assess the importance of health marker information in the decision to smoke cigarettes, and b.) capture the direct effect of smoking, and smoking cessation, on different health outcomes while controlling for unobserved heterogeneity.

Generally, I find that significant reinforcement and withdrawal effects drive smoking dynamics by altering the future marginal utilities of smoking. The reinforcement effect is estimated with a novel construction of past smoking behavior that is consistent with the theoretical notion of a "smoking stock". I empirically construct this stock as a weighted average of several measures of smoking history using using principal components analysis. Consistent with the theory, individuals understand that different smoking choices influence the smoking stock through depreciation and investment coefficients. The smoking stock then reinforces future smoking, through the marginal utility of smoking, and influences health, through the health marker index.

Estimates of the structural parameters suggest that there exists heterogeneity in the effect of the accumulated smoking stock on an index of health markers. Despite this heterogeneity, learning about an individual's own place in the distribution of this effect, at least in the Framingham Heart Study setting, does not appear to significantly inform smokers about the long-term health consequences of smoking. In fact, learning about how smoking effects health markers may actually slightly increase moderate smoking in older individuals. However, individuals that receive sharp, discrete shocks that imply worse health markers typically do scale down their smoking behavior (either by quitting or lowering the intensity with which they smoke). Only when a health marker signal is four times its actual value do simulations suggest individuals curtail their smoking behavior in any significant sense. Therefore, my results are consistent with the literature on personalized health information that have found changes in smoking behavior after serious health shocks (Smith et al., 2001; Sloan et al., 2003; Khwaja et al., 2006; Arcidiacono et al., 2007).

The lack of a change in smoking to marginal changes in health markers, observed from Framingham Heart Study health exams, may be evidence of some limitations of this study. First, while the FHS administers health exams every two to eight years, individuals may be 
observing their health markers at other doctor visits. Interim doctor visits may induce individuals to quit smoking; however, the econometrician would only observe that the individual quit. With respect to the model, the observed quitting would be attributed to the preference error, $\epsilon$. In this case, the conclusion would not be that health markers are uninformative as to the implications of smoking, but rather that the Framingham Heart Study signals of information do not provide any additional information to what is already known. Because of this possibility, I interpret my results on as a lower bound on health marker learning.

Second, as noted in Sloan et al. (2003), smoking behavior may be altered by a change in risk perceptions, which may be changed by new information. Because of the absence of subjective expectation data regarding the effect of smoking on health markers, I am required to place more structure on the learning process. Thus, the assumption of conjugate normal distributions for the signal of information and beliefs may be driving the results. Alternatively, I could specify a beta/binomial learning process. Here, a discrete signal of information (e.g., blood pressure exceeding some threshold) causes individuals to update their continuous belief distribution.However, learning about multiple health marker discrete shocks would be computationally intractable. Furthermore, it is not clear how one could both define the relevant signal (i.e., which health marker) and it's corresponding threshold. I leave different specifications of the learning process for future work.

I find evidence of positive selection with respect to smoking and mortality by estimating the correlation in permanent unobserved heterogeneity between these outcomes. Factor loadings that dictate the effect of the permanent unobserved term on the marginal utility of smoking and on mortality are both estimated to be positive. This finding suggests that individuals that are more likely to smoke, are also more likely to die independently of smoking. Interestingly, the factor loadings that capture the correlation in permanent unobservables across smoking and chronic health and health markers are not statistically different from zero. This finding suggests that, while there exists an unobserved relationship between the propensity to smoking and mortality, the excess in mortality cannot be attributed directly to chronic disease (as defined in this paper) or health markers. However, as noted above, if unobserved factors drive certain individuals to smoke, and smoking predicts the onset of chronic conditions, the unobserved factors may still predict disease related mortality through smoking.

Simulations of the structural model confirm the positive selection and suggest that, when controlling for unobserved heterogeneity, the effects of smoking on mortality outcomes may be less extreme than previously estimated. I find that smoking heavily from age 18 can reduce life expectancy by eight years relative to life-long non-smokers and by four years for light smokers ( $\leq 1$ pack/day) from age 18. I compare my results to those of Taylor et al. (2002); Doll et al. (2004); Brønnum-Hansen et al. (2007) that find overall longevity loss from daily 
smoking to be roughly 7.4-10.5, 10, and 8.7-10.4 years, respectively. Furthermore, quitting smoking by age 30 implies relatively few chronic health or mortality differences, on average, from life-long non-smokers; however, waiting to quit until age 60 implies that the health consequences may be severe. Indeed, as suggested by the literature, the major effects of smoking on health are realized after age 50 (Sloan et al., 2003).

The two main questions of this paper should guide future work. First, are there sources of information, personalized or otherwise, that effectively convince individuals to stop smoking that also are not major health shocks? Do health markers influence smoking behavior in settings other than the Framingham Heart Study? It would be interesting to collect subjective expectation data on risk perceptions in which surveyors explicitly mention individual specific variation in health markers. Would these data show a role for health markers to change risk perceptions and subsequent smoking behavior? Second, what are the sources of unobserved heterogeneity that are shown to be correlated across preferences for smoking and mortality. Surely alcohol consumption may confound smoking and mortality, but what other factors? Finally, would controlling for and modeling parental smoking behavior or parental health outcomes significantly change the role of unobserved heterogeneity in the results of this paper? 


\section{Appendices}

\section{A Solution}

The computational hurdle in calculating the conditional choice probabilities in equation 10 is to solve for the integrated Bellman (EMAX) equation in equation 9. Technically, the EMAX equation must be solved for all possible points $s$ in the state space $S_{i t}$. However, given the long time frame of the model and the mixed discrete/continuous nature of the state space, I employ a variant of the Keane and Wolpin (1994) value function interpolation method for approximating the value function. This method amounts to drawing from the state space, calculating the resulting EMAX function for each draw, and interpolating the EMAX function for all other points. The end goal of this procedure is to generate choice probabilities for each individual $i$, in each time period $t$, conditional on the unobserved heterogeneity $\mu$ and a trial set of the parameters, to enter the likelihood function. My iterative solution method proceeds in two main steps: model simulation and individual specific solution. While the first solution step yields value function regression coefficients from the simulated model, the second step uses these coefficients to calculate the conditional choice probabilities, health marker and smoking stock densities, and chronic health and mortality transition probabilities.

The first step of the solution method is to solve the model for a group of simulated individuals. The goal of this step is to generate a set of regression coefficients that map from the state space to the value function. Because the time horizon is finite $(T=100)$, I can solve the model using backwards induction and I avoid iterating on the value function itself. Starting in the final period $T$, I draw $n$ state vectors and sequences of past smoking behavior $\mathbf{D}_{i T-1}=\left\{d_{i 1}, \ldots, d_{i T-1}\right\} .{ }^{42}$ Each of these $n$ draws represents one simulated individual. For each of the $n$ draws, I construct the main equations of the model for period $T$. Note that because the probability of death at the end of period $T$ equals one, each of the choice specific value functions in period $T$ simply equals the current period utility from the smoking alternative. Next, I posit a relationship between the $n$ calculated value functions and a set of regressors. The regressors include the drawn state variables in addition to interaction and higher-order terms. I then run the regression and generate coefficients that are specific to time period $T$. Next, I repeat the above steps for period $T-1$. When calculating the expected value function in period $T-1$, I use the regression coefficients from period $T$ to approximate the expected future value function. I repeat the above process for all periods back to age seven, $t=7$; that is, I solve the model for all ages between 7 and 100.

The first stage process is conditional on three factors. First, I conduct the simulation

\footnotetext{
${ }^{42}$ In practice I set $n=100$.
} 
above for each possible age at which an individual may have taken her first health exam. ${ }^{43}$ Second, I discretized the support of the unobserved heterogeneity distribution into $K$ points. ${ }^{44}$ For each point $k$ of $\mu$, in addition to each age at the initial health exam, I conduct the above simulation. Thus, I have a full set of value function regression coefficients (from age 7 to 100) for each age at initial health exam and for each unobserved type, $\mu$. Finally, the value function regression coefficients are also conditional on the trial set of parameters used to solve the model.

The second main step, conditional on the same trial set of parameters and using the above regression coefficients, involves solving the model for each individual. For each individual, I solve the model backwards from age 100 to generate conditional choice probabilities, health marker and smoking stock densities, and chronic health and mortality transition probabilities. This process is complicated by the fact that I only have data for the health marker index in some periods.

In each period in which individual $i$ undergoes a health exam, he/she must forecast the future evolution of the state variables and the resulting values associated with all current and future smoking decisions. Luckily however, because the value function regression coefficients approximate the next period value function, I must only construct the expected value of the next period state variables conditional on the current period smoking decision. Because the chronic health and mortality logit probabilities have closed-form expressions, assuming rational expectations makes the next period chronic health and mortality transition expectations straightforward. To forecast the smoking stock and health marker index values one period forward, I use a Monte Carlo method. Conditional on each draw of the Monte Carlo simulator, I construct all other probabilities in the model.

For time periods in which no health exam was taken, in addition to integrating over the future values of the smoking stock and health marker index, I must also integrate over the current period value of the health marker index. In this case, I use the same method as integrating over the future health marker index, only using different draws (from those used to integrate over the future term) of the i.i.d. error term $v$. All other probabilities in the model are constructed conditional on the drawn value of the current period health marker index and averaged.

\footnotetext{
${ }^{43}$ In the data, the ages range from 13 to 62 . As noted above, there exists great variation in the data in the timing of the health exams. However, in the simulation, regardless of age at the initial health exam, I use the average number of years between exams to avoid having to simulate the model for all possible combinations of exam sequences.

${ }^{44}$ In practice I set $K=3$.
} 


\section{B Bayesian Updating}

Here, I derive the posterior beliefs discussed in the main text (Equations 4 and 5). I assume rational expectations such that an individual's initial belief upon entering the sample regarding their true $\theta_{i}$ is the population distribution:

$$
\begin{gathered}
E_{0}\left(\theta_{i}\right)=\tau_{i 0}=\bar{\theta} \\
V_{0}\left(\theta_{i}\right)=\psi_{i 0}=\sigma_{\theta}^{2} .
\end{gathered}
$$

Consider an individual in period $t$ with smoking stock $A_{i t}$. For ease of exposition, assume that an individual takes a health exam each period. When deriving the posterior beliefs in period $t$, an individual considers only her prior beliefs $\left(\tau_{i t-1}, \psi_{i t-1}\right)$ and her signal of information $k_{i t}$. According to Bayes' Rule, the posterior distribution, $f_{t}$, of $\theta_{i}$ is given as:

$$
f_{t}\left(\theta_{i} \mid \kappa_{n}, \tau_{i t-1}, \psi_{i t-1}\right) \propto f_{t-1}\left(\theta_{i}\right) g\left(\kappa_{i t} \mid A_{i t}, \theta_{i}, \sigma_{v}\right) .
$$

Note that while $g\left(\kappa_{i t} \mid A_{i t}, \theta_{i}, \sigma_{v}\right)$ conveys information about $\kappa^{i t}$, an individual knows $A_{i t}$ and, because $\theta_{i}$ is time invariant, can therefore infer information about $\theta_{i}$ over time. This will become more clear in the interpretation of the posterior mean and variance. First consider $g\left(\kappa_{i t} \mid A_{i t}, \theta_{i}, \sigma_{v}\right)$ :

$$
g\left(\kappa_{i t} \mid A_{i t}, \theta_{i}, \sigma_{v}\right)=\frac{1}{\left(2 \pi \sigma_{v}^{2}\right)^{\frac{1}{2}}} \exp \left(\frac{1}{2 \sigma_{v}^{2}}\left(\kappa_{i t}-\theta_{i} A_{i t}\right)^{2}\right) .
$$

Note that because we are concerned with the distribution of $\theta_{i}$, any term that does not include $\theta_{i}$ can be treated as part of the normalizing constant. We can ignore the first term within the parenthesis:

$$
\propto \exp \left(\frac{-1}{2 \sigma_{v}^{2}}\left(-2 \theta_{i} \kappa_{i t} A_{i t}+\theta_{i}^{2} A_{i t}^{2}\right)\right) .
$$

Simplifying and completing the square yields:

$$
\propto \exp \left(-\frac{A_{i t}^{2}}{2 \sigma_{v}^{2}}\left(\theta_{i}-\frac{\kappa_{i t} A_{i t}}{A_{i t}^{2}}\right)^{2}\right) .
$$

Notice that the term subtracted from $\theta_{i}$ is the within (individual $i$ ) variation ordinary least squares estimate of $\theta_{i}$ from the $n^{\text {th }}$ signal of information. Define $\hat{\theta_{i t}}=\frac{\kappa_{i t} A_{i t}}{A_{i t}^{2}}$. Substituting for $\hat{\theta_{i t}}$, we have that:

$$
g\left(\kappa_{i t} \mid A_{i t}, \theta_{i}, \sigma_{v}\right) \propto \exp \left(-\frac{A_{i t}^{2}}{2 \sigma_{v}^{2}}\left(\theta_{i}-\hat{\theta_{i t}}\right)^{2}\right) .
$$

Now consider the prior probability distribution of $\theta_{i}$ :

$$
f_{t-1}\left(\theta_{i}\right)=\frac{1}{\left(2 \pi \psi_{i t-1}\right)^{\frac{1}{2}}} \exp \left(\frac{1}{2 \psi_{i t-1}}\left(\theta_{i}-\tau_{i t-1}\right)^{2}\right) .
$$


The nice aspect of the conjugate distribution assumption is that we can characterize the posterior distribution sufficiently with closed form expressions for the posterior mean and variance. Therefore, we only have to characterize that part of the posterior density that captures the mean and variance. In that light, consider the product of the exponential portions of Equations B.3 and B.4 after rearranging terms and absorbing those without $\theta_{i}$ into the normalizing constant:

$$
f_{t}\left(\theta_{i}\right) \propto\left(-\frac{1}{2 \psi_{i t-1} \sigma_{v}^{2}}\left(\theta_{i}^{2}\left(A_{i t}^{2} \psi_{i t-1}+\sigma_{v}^{2}\right)-2 \theta_{i}\left(A_{i t}^{2} \psi_{i t-1} \hat{\theta}_{i t}+\sigma_{v}^{2} \tau_{i t-1}\right)\right)\right) .
$$

After rearranging and completing the square, we have the kernel of a normal distribution representing the posterior distribution:

$$
f_{t}\left(\theta_{i}\right) \propto\left(-\frac{A_{i t}^{2} \psi_{i t-1}+\sigma_{v}^{2}}{2 \psi_{i t-1} \sigma_{v}^{2}}\left(\theta_{i}-\left(\frac{A_{i t}^{2} \psi_{i t-1} \hat{\theta}_{i t}+\sigma_{v}^{2} \tau_{i t-1}}{A_{i t}^{2} \psi_{i t-1}+\sigma_{v}^{2}}\right)^{2}\right)\right) .
$$

The posterior mean and variance is:

$$
\begin{gathered}
\tau_{i t}=E\left(\theta_{i} \mid \kappa_{t}, \tau_{i t-1}, \psi_{i t-1}\right)=\left(\frac{A_{i t}^{2} \psi_{i t-1}}{A_{i t}^{2} \psi_{i t-1}+\sigma_{v}^{2}}\right) \hat{\theta}_{i t}+\left(\frac{\sigma_{v}^{2}}{A_{i t}^{2} \psi_{i t-1}+\sigma_{v}^{2}}\right) \tau_{i t-1} \\
\psi_{t}=\operatorname{Var}\left(\theta_{i} \mid \psi_{i t-1}, \sigma_{v}\right)=\frac{\psi_{i t-1} \sigma_{v}^{2}}{A_{i t}^{2} \psi_{i t-1}+\sigma_{v}^{2}}
\end{gathered}
$$

Rearranging these equations yields the posterior mean and variance equations above.

\section{References}

Ackerberg, D. (2003). Advertising, Learning, and Consumer Choice in Experience Good Markets: An Empirical Examination. International Economic Review, 44 (3), 1007-1040.

Adda, J. and Lechene, V. (2001). Smoking and Endogenous Mortality: Does Heterogeneity in Life Expectancy Explain Differences in Smoking Behavior, discussion Paper Series, Department of Economics, University of Oxford, ISSN 1471-0498.

— and - (2004). On the identification of the effect of smoking on mortality, discussion Paper Series, Department of Economics, University of Oxford, Working Paper Number 184.

Aguirregabiria, V. and Mira, P. (2010). Dynamic discrete choice structural models: A survey. Journal of Econometrics, 156 (1), 38-67. 
Arcidiacono, P., Sieg, H. and Sloan, F. A. (2007). Living Rationally Under the Volcano? An Empirical Analysis of Heavy Drinking and Smoking. International Economic Review, 48 (1), $37-65$.

Becker, G. S. and Murphy, K. (1988). A Theory of Rational Addiction. Journal of Political Economy, 96 (4), 675-700.

Bennett, C. H. and Richardson, D. R. (1984). Effects of Chronic Tobacco Smoke Exposure on Arterial Blood Pressure Regulation. American Journal of Physiology: Heart and Circulatory Physiology, 247.

Bernheim, B. D. and Rangel, A. (2004). Addiction and cue-triggered decision processes. American Economic Review, 94 (5), 1558-1590.

Blau, D. M. and Gilleskie, D. B. (2008). The Role of Reitree Health Insurance in the Employment Behavior of Older Men. International Economic Review, 49 (2), 475-514.

Brønnum-Hansen, H., Juel, K., Davidsen, M. and Rensen, J. S. (2007). Impact of selected risk factors on expected lifetime without long-standing limiting illness in denmark. Preventive Medicine, 45, 49-53.

Carbone, J. C., Kverndokk, S. and Rogeberg, O. J. (2005). Smoking, Health, Risk, and Perception. Journal of Health Economics, 24, 631-653.

Chan, T. Y. and Hamilton, B. (2006). Learning, Private Information, and the Economic Evaluation of Randomized Experiments. Journal of Political Economy, 114 (6), 997-1040.

Chernew, M., Gowrisankaran, G. and Scanlon, D. P. (2008). Learning and the Value of Information: Evidence from Health Plan Report Cards. Journal of Econometrics, 144, 156-174.

Crawford, G. S. and Shum, M. (2005). Uncertainty and Learning in Pharmaceutical Demand. Econometrica, 73 (4), 1137-1173.

Doll, R., Peto, R., Boreham, J., Gray, R. and Sutherland, I. (2004). Mortality in Relation to Smoking: 50 Years' Observations on Male British Doctors. British Medical Journal, 328, 1519-1528.

—, - , Wheatley, K., Gray, R. and Sutherland, I. (1994). Mortality in Relation to Smoking: 40 Years' Observations on Male British Doctors. British Medical Journal, 309, 901-911. 
Epstein, L. G. and Zin, S. E. (1991). Substitution, Risk Aversion, and the Temporal Behavior of Consumption and Asset Returns: An Empirical Analysis. The Journal of Political Economy, 99 (2).

Garrison, R., Kannel, W., Feinleib, M., Castelli, W., McNamara, P. and Padgett, S. (1978). Cigarette Smoking and HDL Cholesterol. Atherosclerosis, 30, 17-25.

Gilleskie, D. B. (1998). A Dynamic Stochastic Model of Medical Care Use and Work Absence. Econometrica, 66 (1), 1-45.

Gruber, J. and Koszegi, B. (2001). Is Addiction "Rational"? Theory and Evidence. The Quarterly Journal of Economics, 116 (4).

Heckman, J. J. and Singer, B. (1984). A Method for Minimizing the Impact of Distributional Assumptions in Econometric Models for Duration Data. Econometrica, 52 (2), 271-320.

Keane, M. P. and Wolpin, K. I. (1994). The Solution and Estimation of Discrete Choice Dynamic Programming Models by Simulation and Interpolation: Monte Carlo Evidence. The Review of Economics and Statistics, 76 (4).

KhwajA, A. (2010). A Life Cycle Analysis of the Effects of Medicare on Individual Health Incentives and Health Outcomes. Journal of Econometrics, 156, 130-147.

-, Sloan, F. A. and Chung, S. (2006). Learning About Individual Risk and the Decision to Smoke. International Journal of Industrial Organization, 24 (), 683-699.

Kreps, D. M. and Porteus, E. L. (1978). Temporal Resolution of Uncertainty and Dynamic Choice Theory. Econometrica, 46 (1).

— and - (1979). Dynamic Choice Theory and Dynamic Programming. Econometrica, 47 (1).

MirA, P. (2007). Uncertain Infant Mortality, Learning, and Life-Cycle Fertility. International Economic Review, 48 (3), 809-846.

Mroz, T. (1999). Discrete Factor Approximations in Simultaneous Equation Models: Estimating the Impact of a Dummy Endogenous Variable on a Continuous Outcome. Journal of Econometrics, 92 (2), 233-274.

Oмviк, P. (1996). How Smoking Affects Blood Pressure. Blood Pressure, 5, 71-77. 
Orphanides, A. and Zervos, D. (1995). Rational Addiction with Learning and Regret. Journal of Political Economy, 103 (4), 739-758.

Rust, J. (1987). Optimal Replacement of Gmc Bus Engines: An Empirical Model of Harold Zurcher. Econometrica, 55 (5), 999-1033.

Sickles, R. C. and Williams, J. (2008). Turning from Crime: A Dynamic Perspective. Journal of Econometrics, 145, 158-173.

Sloan, F. A., Smith, V. K. and Taylor, D. H. (2003). The Smoking Puzzle: Information, Risk Perception, and Choice. Harvard University Press.

Smith, V. K., Taylor, D. H., Sloan, F. A., Johnson, F. R. and Desvousges, W. H. (2001). Do Smokers Respond to Health Shocks? The Review of Economics and Statistics, 83 (4), 675687.

Suranovic, S. M., Goldarb, R. S. and Leonard, T. C. (1999). An Economic Theory of Cigarette Addiction. Journal of Health Economics, 18, 1-29.

Taylor, D. H., Hasselblad, V., Henley, S. J., Thun, M. J. and Sloan, F. A. (2002). Benefits of Smoking Cessation for Longevity. American Journal of Public Health, 92 (6).

United States Department of Health and Human Services (1990). The Health Benefits of Smoking Cessation: A Report of the Surgeon General's Report.

Viscusi, W. K. (1990). Do Smokers Underestimate Risks? Journal of Political Economy, 98 (6), 1253-1269.

- and Evans, W. N. (1990). Utility Functions that Depend on Health Status: Estimates and Economics Implications. American Economic Review, 80 (3), 353-374.

- and Hakes, J. K. (2008). Risk Beliefs and Smoking Behavior. Economic Inquiry, 46 (1), $45-59$.

Vyas, S. and Kumaranayake, L. (2006). Constructing Socio-Economic Status Indices: How to Use Principal Component Analysis. Oxford University Press in association with the London School of Hygiene and Tropical Medicine. 


\title{
Smoking, Expectations, and Health: A Dynamic Stochastic Model of Lifetime Smoking Behavior
}

Figures

\author{
Michael Darden* \\ Tulane University $*$
}

October 12, 2011

\section{Figures}

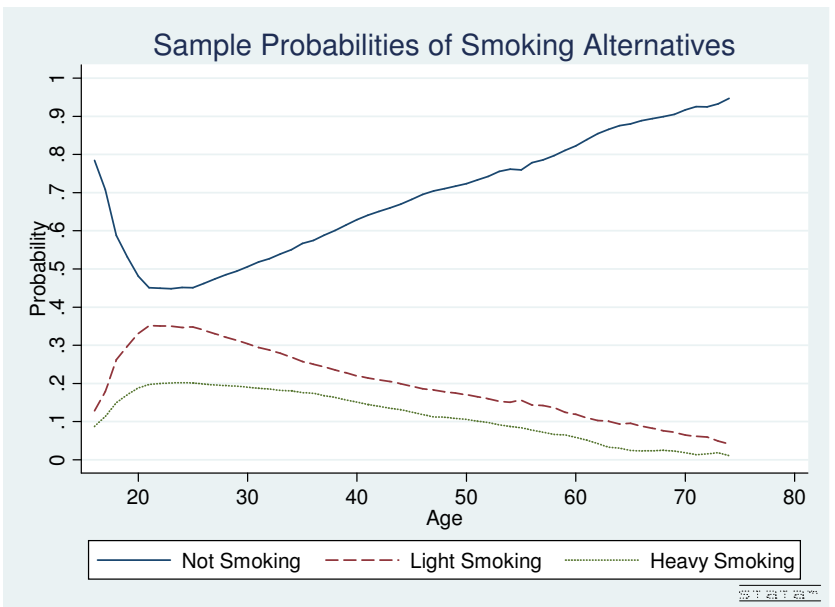

Figure 1: Sample Choice Probabilities by Age

\footnotetext{
*Email:mdarden1@tulane.edu

The Framingham Offspring Study (FOS) is conducted and supported by the NHLBI in collaboration with the FOS Study Investigators. This manuscript was prepared using a limited access dataset obtained from the NHLBI and does not necessarily reflect the opinions or views of the FOS or the NHLBI.

Department of Economics, 206 Tilton Memorial Hall, Tulane University, New Orleans, LA 70118
} 


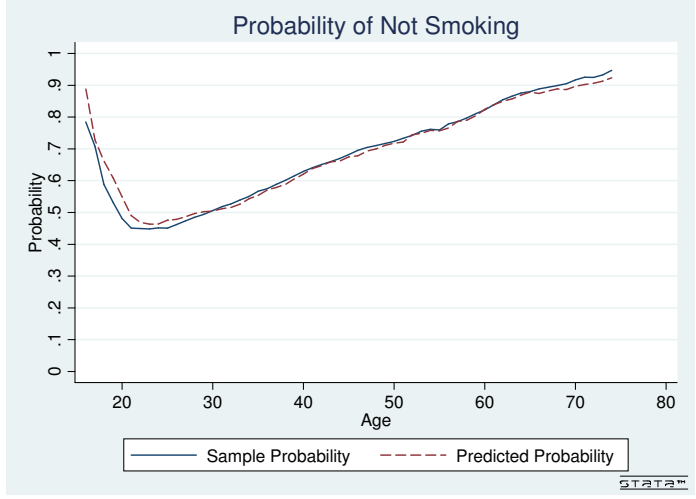

a.

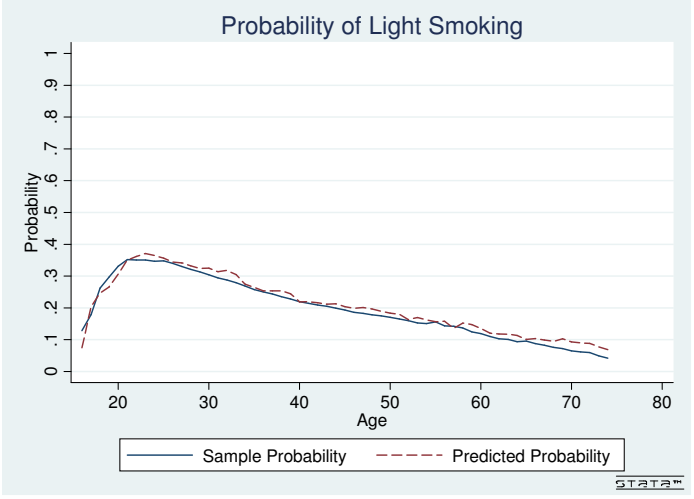

b.

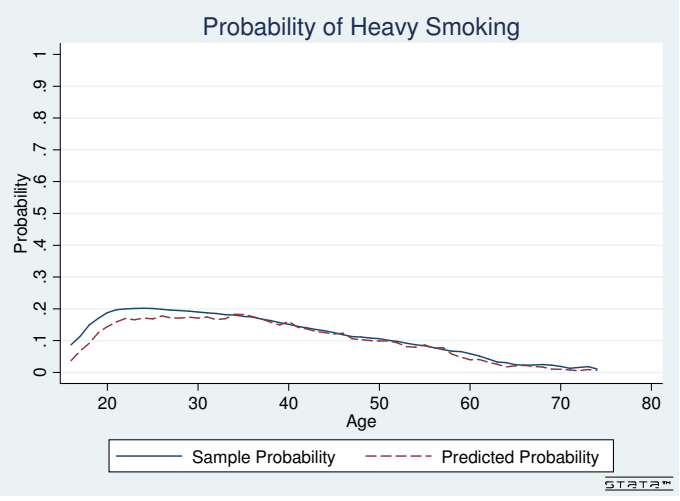

c.

Figure 2: Smoking Behavior by Age: Predicted and Sample Probabilities 


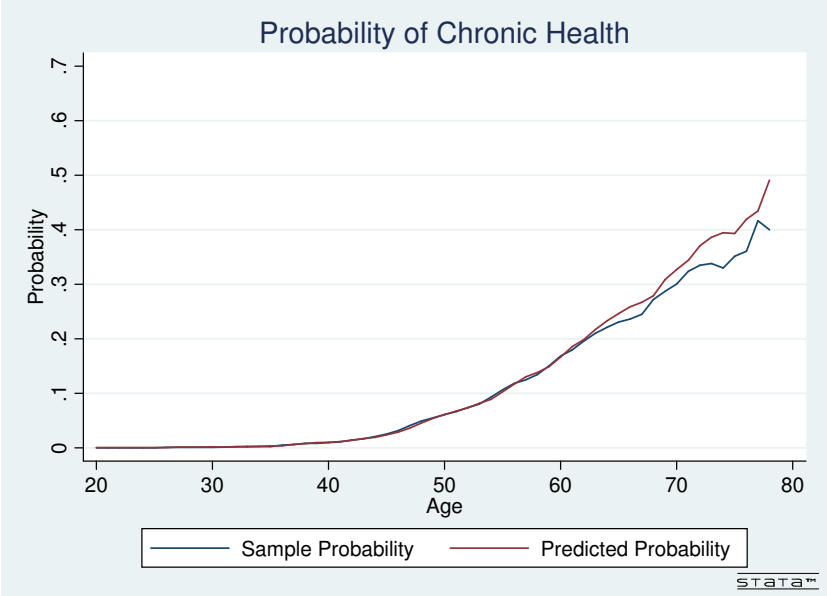

Figure 3: Chronic Health State by Age: Predicted and Sample Probabilities 


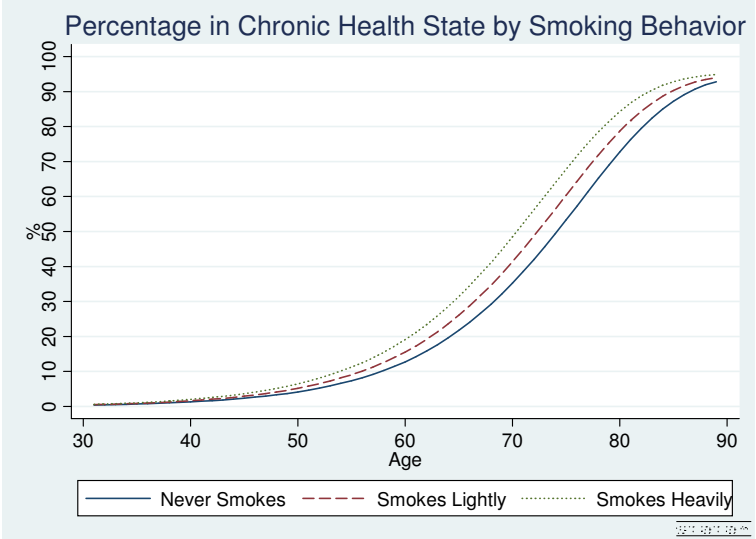

a.

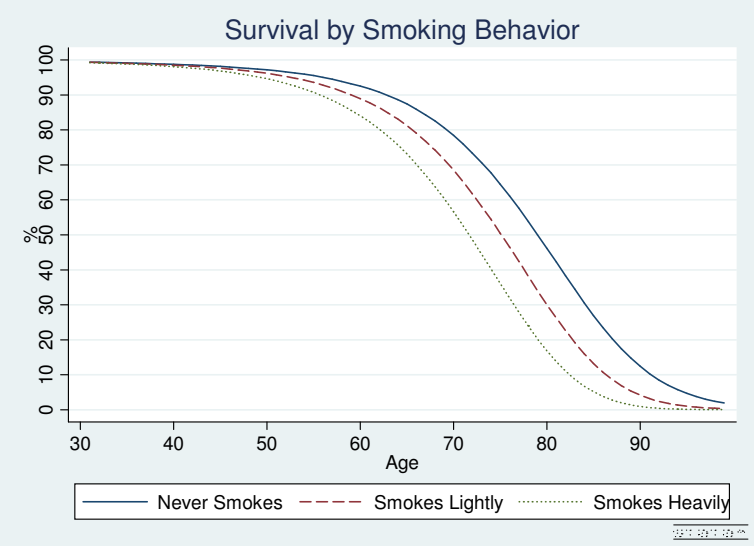

b.

Figure 4: Percentage of simulated sample a.) in the chronic health state and b.) remaining, by age and quit status 


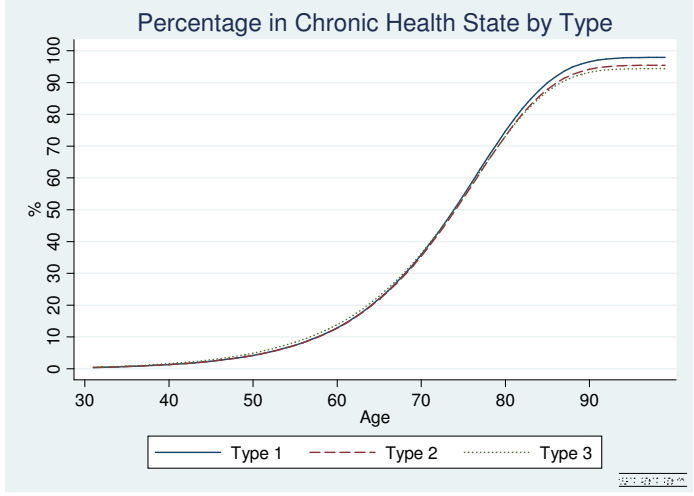

a.

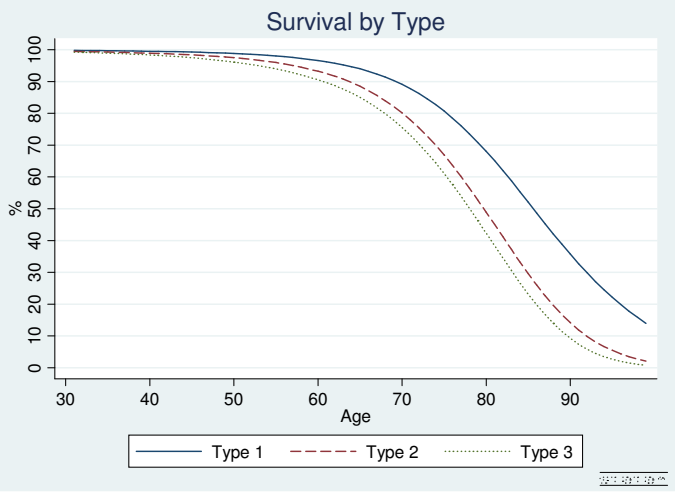

b.

Figure 5: a.) Simulated chronic health state by age at baseline. b.) Simulated longevity by age at baseline. c.) Simulated chronic health state by age assuming no smoking. d.) Simulated longevity by age assuming no smoking. 


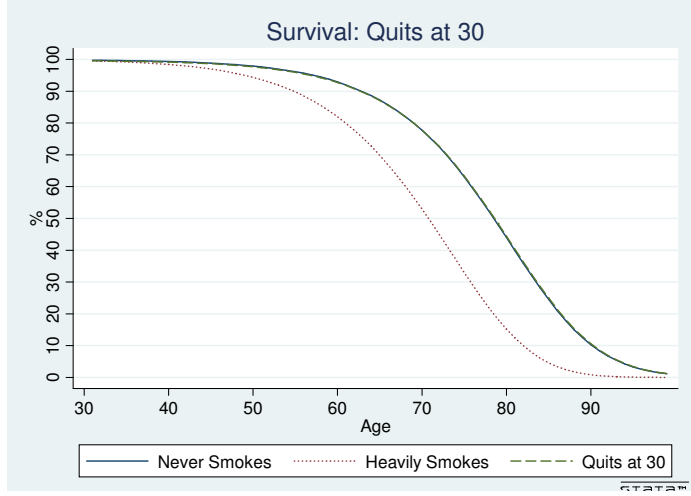

a.

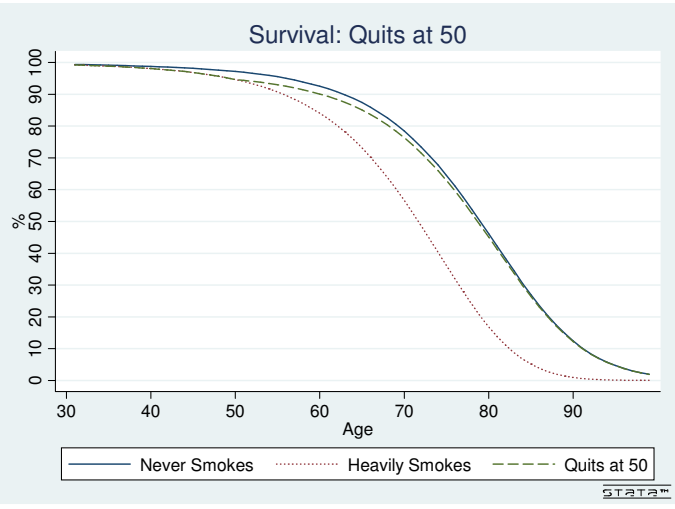

c.

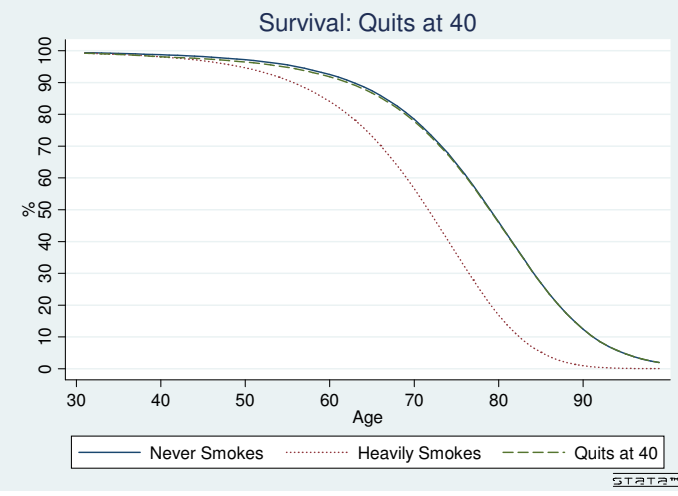

b.

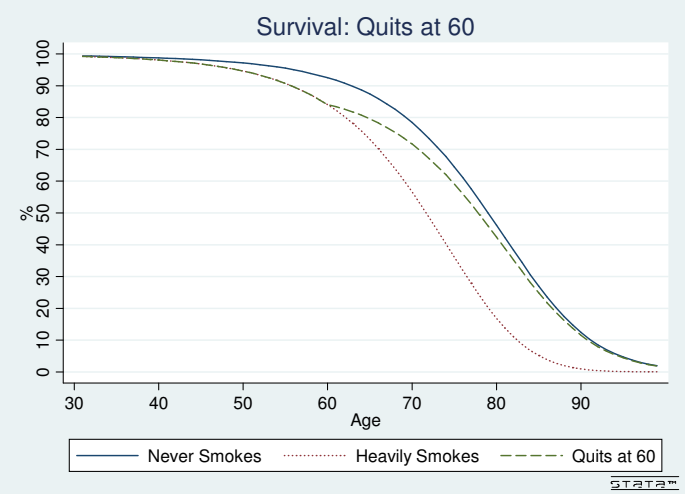

d.

Figure 6: Percentage of simulated sample remaining, by age and quit status

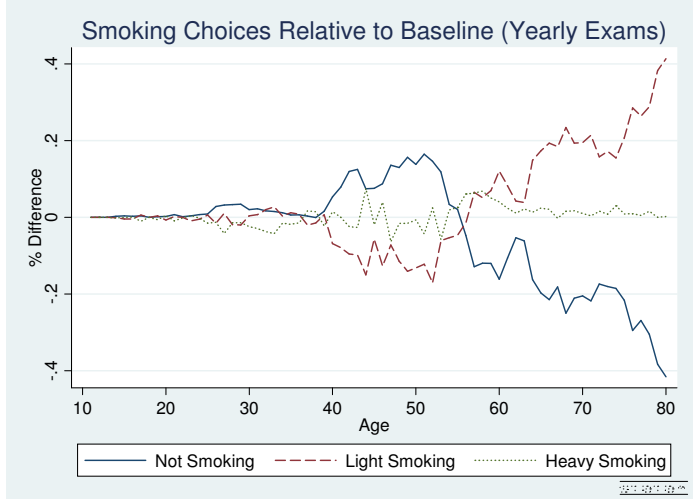

a.

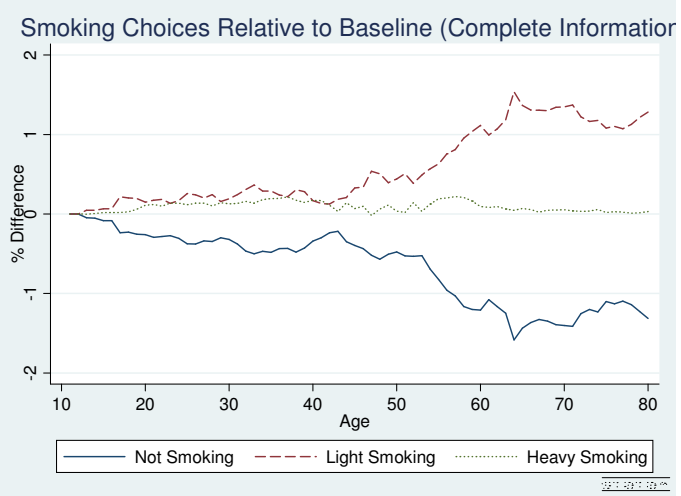

b.

Figure 7: Average difference in smoking probabilities, relative to baseline choices, by age and across different policy scenarios 


\title{
Smoking, Expectations, and Health: A Dynamic Stochastic Model of Lifetime Smoking Behavior
}

Tables

\author{
Michael Darden* \\ Tulane University $*$
}

October 12, 2011

\section{Tables}

Table 1: Sample Construction

\begin{tabular}{ll}
\hline \hline $\mathbf{N}$ & Description \\
\hline 4989 & Framingham Heart Survey Offspring Cohort Participants - Restricted Sample \\
3730 & Sample after dropping those individuals that skipped one or more of the health exams \\
3008 & Sample after dropping all person/year observations of individuals who attrit \\
\hline 3008 unique individuals yields 19461 person/year observations.
\end{tabular}

\footnotetext{
*Email:mdarden1@tulane.edu

The Framingham Offspring Study (FOS) is conducted and supported by the NHLBI in collaboration with the FOS Study Investigators. This manuscript was prepared using a limited access dataset obtained from the NHLBI and does not necessarily reflect the opinions or views of the FOS or the NHLBI.

Department of Economics, 206 Tilton Memorial Hall, Tulane University, New Orleans, LA 70118
} 
Table 2: Sample Characteristics by Exam

\begin{tabular}{c|cccccc}
\hline \hline Exam & Mean Year & Mean Age & St. Dev. Age & \% Female & \% Married & \# Individuals \\
\hline 1 & 1973 & 37.0 & $(10.28)$ & 50.0 & 80.5 & 3008 \\
2 & 1981 & 44.3 & $(10.05)$ & 50.1 & 82.9 & 2921 \\
3 & 1985 & 48.3 & $(9.99)$ & 51.1 & 83.0 & 2849 \\
4 & 1988 & 51.5 & $(9.99)$ & 51.5 & 80.6 & 2796 \\
5 & 1992 & 55.0 & $(9.83)$ & 52.1 & 79.9 & 2709 \\
6 & 1996 & 58.6 & $(9.69)$ & 52.7 & 77.2 & 2613 \\
7 & 1999 & 61.5 & $(9.58)$ & 53.1 & 74.7 & 2565 \\
\hline
\end{tabular}

Ages in the sample range from 13 in exam 1 to 88 in exam 7.

Table 3: Education

\begin{tabular}{cc}
\hline $\begin{array}{c}\text { Education } \\
\text { Years }\end{array}$ & $\begin{array}{c}\text { \% of } \\
\text { Sample }\end{array}$ \\
\hline $0-4$ & $3.2 \%$ \\
$5-8$ & 1.0 \\
$9-11$ & 6.1 \\
12 & 32.8 \\
$13-16$ & 43.2 \\
$17+$ & 13.8 \\
\hline$N=3008$. Percentages reflect \\
highest attained level of education.
\end{tabular}

Table 4: Smoking Behavior by Exam

\begin{tabular}{c|ccc}
\hline \hline Exam & Nonsmokers & $\begin{array}{c}\text { Light Smokers } \\
\text { 1 Pack/Day }\end{array}$ & $\begin{array}{c}\text { Heavy Smokers } \\
\text { > 1 Pack/Day }\end{array}$ \\
\hline 1 & $59.0 \%$ & $26.7 \%$ & $14.3 \%$ \\
2 & 61.3 & 24.4 & 14.2 \\
3 & 77.2 & 14.3 & 8.5 \\
4 & 81.2 & 12.8 & 6.0 \\
5 & 85.2 & 11.0 & 3.9 \\
6 & 87.9 & 9.5 & 2.6 \\
7 & 88.9 & 8.7 & 2.5 \\
\hline
\end{tabular}


Table 5: Chronic Health by Exam

\begin{tabular}{c|cc}
\hline \hline Exam & $\begin{array}{c}\text { Chronic Condition } \\
\text { at Exam }\end{array}$ & $\begin{array}{c}\text { Newly Chronically Ill } \\
\text { at Exam }\end{array}$ \\
\hline 1 & $0.2 \%$ & $0.0 \%$ \\
2 & 4.0 & 3.8 \\
3 & 7.0 & 3.4 \\
4 & 9.6 & 3.1 \\
5 & 12.2 & 3.9 \\
6 & 16.0 & 5.0 \\
7 & 20.3 & 4.8 \\
\hline
\end{tabular}

Table 6: Smoking Summary Statistics (Conditional on Ever Smoking)

\begin{tabular}{l|cccc}
\hline \hline & Mean (Median) & S.D. & Min & Max \\
\hline First Age Smoking & $19.57(18)$ & 7.45 & 7 & 67 \\
Total Years Smoking & $24.78(24)$ & 14.07 & 1 & 68 \\
Tenure Smoking (Years) & $21.13(19)$ & 14.85 & 1 & 68 \\
Last Age Smoking & $44.76(45)$ & 12.84 & 13 & 76 \\
$\quad$ & & & & \\
\hline
\end{tabular}


Table 7: Observed Transitions

\begin{tabular}{|c|c|c|c|c|c|c|}
\hline \multirow{3}{*}{$\begin{array}{l}\text { Behavior One } \\
\text { Period Prior }\end{array}$} & \multicolumn{3}{|c|}{ Behavior One Period Post } & \multicolumn{3}{|c|}{ Behavior Three Periods Post } \\
\hline & $\begin{array}{l}\text { Not } \\
\text { Smoking }\end{array}$ & Light & $\begin{array}{l}\text { Heavy } \\
\text { Smokino }\end{array}$ & Not & Light & Heavy \\
\hline & Smoking & Smoking & Smoking & Smoking & Smoking & Smoking \\
\hline Not Smoking & $95.86 \%$ & $2.93 \%$ & $1.22 \%$ & $91.72 \%$ & $5.76 \%$ & $2.51 \%$ \\
\hline Light Smoking & 9.21 & 89.29 & 1.51 & 17.35 & 79.81 & 2.84 \\
\hline Heavy Smoking & 5.66 & 2.78 & 91.56 & 11.12 & 5.31 & 83.58 \\
\hline \multicolumn{7}{|c|}{ Transitions Around Health Exams } \\
\hline Not Smoking & 96.38 & 3.20 & 0.42 & 97.00 & 2.55 & 0.45 \\
\hline Light Smoking & 21.40 & 70.29 & 8.30 & 29.45 & 63.50 & 7.06 \\
\hline Heavy Smoking & 14.50 & 16.47 & 69.03 & 20.62 & 16.48 & 62.90 \\
\hline \multicolumn{7}{|c|}{ Transitions Around Chronic Health Shocks } \\
\hline Not Smoking & 98.94 & 0.89 & 0.18 & 97.18 & 2.59 & 0.24 \\
\hline Light Smoking & 24.78 & 73.45 & 1.77 & 39.36 & 54.26 & 6.38 \\
\hline Heavy Smoking & 21.43 & 8.33 & 70.24 & 39.71 & 13.24 & 47.06 \\
\hline
\end{tabular}

Table 8: Health Marker Index Weights

\begin{tabular}{lr}
\hline \hline Variable & Weight \\
\hline Systolic Blood Pressure & 0.657 \\
Diastolic Blood Pressure & 0.637 \\
Total Cholesterol & 0.306 \\
High-Density Lipoprotein (HDL) & -0.177 \\
Diabetes & 0.193 \\
\hline
\end{tabular}

Table 9: Smoking Stock Weights

\begin{tabular}{lr}
\hline \hline Variable & Weight \\
\hline Experience & 0.309 \\
Duration & 0.589 \\
Cessation & -0.517 \\
Intensity & 0.540 \\
\hline
\end{tabular}


Table 10: Variation in Exam Timing

\begin{tabular}{c|cccccc}
\hline \hline Years & Exam & & & & & \\
Between Exams & $\mathbf{2}$ & $\mathbf{3}$ & $\mathbf{4}$ & $\mathbf{5}$ & $\mathbf{6}$ & $\mathbf{7}$ \\
\hline 1 & 0 & 0 & 4 & 0 & 0 & 103 \\
2 & 0 & 27 & 63 & 36 & 49 & 718 \\
3 & 0 & 141 & 1659 & 358 & 326 & 958 \\
4 & 0 & 1951 & 1000 & 2242 & 2019 & 682 \\
5 & 6 & 657 & 57 & 55 & 184 & 90 \\
6 & 27 & 65 & 12 & 16 & 28 & 13 \\
7 & 1500 & 8 & 1 & 2 & 7 & 1 \\
8 & 1302 & 0 & 0 & 0 & 0 & 0 \\
9 & 64 & 0 & 0 & 0 & 0 & 0 \\
10 & 22 & 0 & 0 & 0 & 0 & 0 \\
Total & 2921 & 2849 & 2796 & 2709 & 2613 & 2565 \\
\hline
\end{tabular}


Table 11: Main Parameter Estimates

\begin{tabular}{|c|c|c|c|c|}
\hline Description & Chronic Condition & Parameter & Estimate & $\overline{\mathrm{ASE}}$ \\
\hline \multicolumn{5}{|l|}{ Utility Parameters } \\
\hline \multicolumn{5}{|l|}{ Constants } \\
\hline & No & $\alpha_{00}$ & 26.039 & 2.261 \\
\hline & Yes & $\alpha_{01}$ & 1.884 & 0.345 \\
\hline \multicolumn{5}{|l|}{ Consumption - Light Smoking } \\
\hline Constant & No & $\alpha_{10}$ & -6.191 & 0.110 \\
\hline Consumption*Smoking Stock & No & $\alpha_{20}$ & 0.001 & 0.000 \\
\hline Consumption*Health Marker Index & No & $\alpha_{30}$ & 0.000 & 0.000 \\
\hline Consumption*Age & No & $\alpha_{40}$ & 0.075 & 0.001 \\
\hline Consumption & Yes & $\alpha_{11}$ & -5.675 & 0.160 \\
\hline Consumption*Smoking Stock & Yes & $\alpha_{21}$ & 2.326 & 0.018 \\
\hline Consumption*Health Marker Index & Yes & $\alpha_{31}$ & -0.005 & 0.002 \\
\hline Consumption*Age & Yes & $\alpha_{41}$ & 0.002 & 0.001 \\
\hline \multicolumn{5}{|l|}{ Consumption - Heavy Smoking } \\
\hline Constant & No & $\alpha_{50}$ & -18.810 & 0.036 \\
\hline Consumption*Smoking Stock & No & $\alpha_{60}$ & 1.686 & 0.013 \\
\hline Consumption*Health Marker Index & No & $\alpha_{70}$ & -0.001 & 0.000 \\
\hline Consumption*Age & No & $\alpha_{80}$ & 0.000 & 0.000 \\
\hline Consumption & Yes & $\alpha_{51}$ & 0.011 & 0.004 \\
\hline Consumption*Smoking Stock & Yes & $\alpha_{61}$ & 2.665 & 0.018 \\
\hline Consumption*Health Marker Index & Yes & $\alpha_{71}$ & 0.001 & 0.000 \\
\hline Consumption*Age & Yes & $\alpha_{81}$ & -0.102 & 0.004 \\
\hline \multicolumn{5}{|l|}{ Withdrawal } \\
\hline & Yes & $\alpha_{91}$ & -0.938 & 0.123 \\
\hline \multicolumn{5}{|l|}{ Smoking Stock } \\
\hline & No & $\alpha_{100}$ & -0.025 & 0.008 \\
\hline & Yes & $\alpha_{101}$ & 1.488 & 0.053 \\
\hline \multicolumn{5}{|l|}{ Smoking Stock Squared } \\
\hline & No & $\alpha_{110}$ & -0.001 & 0.001 \\
\hline & Yes & $\alpha_{111}$ & -0.462 & 0.005 \\
\hline \multicolumn{5}{|l|}{ Learning Parameters } \\
\hline Mean Effect & & $\bar{\theta}$ & 0.002 & 0.000 \\
\hline Standard Deviation of $\theta_{i}$ & & $\sigma_{\theta}$ & 0.061 & 0.002 \\
\hline Standard Deviation of $v$ & & $\sigma_{v}$ & 1.043 & 0.004 \\
\hline \multicolumn{5}{|l|}{ Additional Health Marker Index Parameters } \\
\hline Lagged Health Marker Index & & $\zeta$ & 0.796 & 0.001 \\
\hline Age in Years & & $\phi_{1}$ & 0.006 & 0.000 \\
\hline Female & & $\phi_{2}$ & -0.121 & 0.003 \\
\hline Education in Years & & $\phi_{3}$ & -0.068 & 0.001 \\
\hline Married & & $\phi_{4}$ & 0.000 & 0.000 \\
\hline Constant & & $\phi_{5}$ & 1.027 & 0.013 \\
\hline
\end{tabular}


Table 12: Other Parameter Estimates

\begin{tabular}{|c|c|c|c|}
\hline Description & Parameter & Estimate & ASE \\
\hline \multicolumn{4}{|l|}{ Smoking Stock Parameters } \\
\hline Depreciation Rate & $\delta_{1}$ & 0.421 & 0.002 \\
\hline Investment, Light Smoking & $\delta_{2}$ & 0.344 & 0.002 \\
\hline Investment, Heavy Smoking & $\delta_{2}$ & 0.427 & 0.002 \\
\hline Standard Deviation of $\eta$ & $\sigma_{\eta}$ & 0.135 & 0.000 \\
\hline \multicolumn{4}{|l|}{ Chronic Health Parameters } \\
\hline Constant & $\lambda_{0}$ & -12.040 & 0.074 \\
\hline Health Marker Index & $\lambda_{1}$ & 0.254 & 0.021 \\
\hline Health Marker Index Squared & $\lambda_{2}$ & -0.014 & 0.001 \\
\hline 1980s*Health Marker Index & $\lambda_{3}$ & 0.000 & 0.000 \\
\hline 1990s*Health Marker Index & $\lambda_{4}$ & 0.000 & 0.000 \\
\hline Choice & $\lambda_{5}$ & 0.303 & 0.024 \\
\hline Choice*Health Marker Index & $\lambda_{6}$ & -0.011 & 0.002 \\
\hline Age & $\lambda_{7}$ & 0.116 & 0.002 \\
\hline Education & $\lambda_{8}$ & 0.007 & 0.001 \\
\hline Gender & $\lambda_{9}$ & 0.012 & 0.004 \\
\hline Married & $\lambda_{10}$ & -0.063 & 0.013 \\
\hline \multicolumn{4}{|l|}{ Mortality Parameters } \\
\hline Constant & $\omega_{0}$ & -8.805 & 0.010 \\
\hline Health Marker Index & $\omega_{1}$ & 0.000 & 0.000 \\
\hline Health Marker Index Squared & $\omega_{2}$ & 0.001 & 0.000 \\
\hline Chronic Health State & $\omega_{3}$ & 4.075 & 0.091 \\
\hline Choice & $\omega_{4}$ & 0.000 & 0.000 \\
\hline Choice*Health Marker Index & $\omega_{5}$ & 0.007 & 0.001 \\
\hline Choice*Chronic Health State & $\omega_{6}$ & 0.516 & 0.028 \\
\hline 1980s* Chronic Health State & $\omega_{7}$ & -0.057 & 0.015 \\
\hline 1990s* Chronic Health State & $\omega_{8}$ & -0.268 & 0.037 \\
\hline Age & $\omega_{9}$ & 0.045 & 0.002 \\
\hline Gender & $\omega_{10}$ & -0.050 & 0.013 \\
\hline Education & $\omega_{11}$ & -0.101 & 0.006 \\
\hline Married & $\omega_{12}$ & -0.260 & 0.041 \\
\hline \multicolumn{4}{|l|}{ Heterogeneity Parameters } \\
\hline \multicolumn{4}{|l|}{ Utility: No Chronic Condition } \\
\hline Not Smoking & $\rho_{u 00}$ & 0.064 & 0.020 \\
\hline Light Smoking & $\rho_{u 01}$ & 2.678 & 0.145 \\
\hline Heavy Smoking & $\rho_{u 02}$ & 8.627 & 0.101 \\
\hline \multicolumn{4}{|l|}{ Utility: Chronic Condition } \\
\hline Not Smoking & $\rho_{u 10}$ & 2.052 & 0.121 \\
\hline Light Smoking & $\rho_{u 11}$ & -0.106 & 0.033 \\
\hline Heavy Smoking & $\rho_{u 12}$ & 0.113 & 0.033 \\
\hline Stock & $\rho_{A}$ & 0.653 & 0.002 \\
\hline Health Marker Index & $\rho_{R}$ & 0.000 & 0.000 \\
\hline Chronic Health & $\rho_{H}$ & 0.001 & 0.001 \\
\hline Mortality & $\rho_{M}$ & 1.022 & 0.137 \\
\hline \multicolumn{4}{|l|}{ Mass Points and Probabilities } \\
\hline Mass Point 1 & $\mu_{1}$ & 0.000 & - \\
\hline Mass Point 2 & $\mu_{2}$ & 1.286 & 0.007 \\
\hline Mass Point 3 & $\mu_{2}$ & 1.000 & - \\
\hline Coef. Weight on Mass Point 1 & $\theta_{1}$ & -2.625 & 0.778 \\
\hline Coef. Weight on Mass Point 2 & $\theta_{2}$ & -1.296 & 0.220 \\
\hline \multicolumn{4}{|l|}{ Miscellaneous Parameters } \\
\hline Discount Factor & $\beta$ & 0.950 & - \\
\hline Log-Likelihood Value & $L(\Theta)$ & -31580.682 & \\
\hline
\end{tabular}

Mass points 1 and 3 are fixed at 0 and 1 respectively. Mass point 2 is estimated and its location is $\frac{\exp (1.286)}{1+\exp (1.286)}=0.783$. The corresponding probabilities of mass points 1 through 3 are $0.054,0.204$, and 0.742 . 
Table 13: Model Fit: Choice Probabilities

\begin{tabular}{|c|c|c|c|c|c|c|}
\hline \multirow[b]{2}{*}{ Exam } & \multicolumn{2}{|c|}{ Not Smoking } & \multicolumn{2}{|c|}{ Light Smoking } & \multicolumn{2}{|c|}{ Heavy Smoking } \\
\hline & Predicted & Observed & Predicted & Observed & Predicted & Observed \\
\hline \multicolumn{7}{|c|}{ Unconditional on Chronic Health State. \# Person/Year Obs. =19,461 } \\
\hline 1 & 61.11 & 59.01 & 25.09 & 26.70 & 13.80 & 14.30 \\
\hline 2 & 68.66 & 61.35 & 19.65 & 24.44 & 11.69 & 14.21 \\
\hline 3 & 71.50 & 77.22 & 18.43 & 14.25 & 10.07 & 8.53 \\
\hline 4 & 78.08 & 81.22 & 14.74 & 12.77 & 7.18 & 6.01 \\
\hline 5 & 81.96 & 85.16 & 12.54 & 10.96 & 5.50 & 3.88 \\
\hline 6 & 86.20 & 87.87 & 9.87 & 9.53 & 3.93 & 2.60 \\
\hline 7 & 89.48 & 88.89 & 7.62 & 8.65 & 2.90 & 2.46 \\
\hline Mean & 76.71 & 77.25 & 15.42 & 15.33 & 7.87 & 7.43 \\
\hline
\end{tabular}

Conditional on No Chronic Condition, $\left(H_{i t}=0\right)$. \# Person/Year Obs. $=17,601$

$\begin{array}{crrrrrr}1 & 61.12 & 58.99 & 25.13 & 26.72 & 13.76 & 14.29 \\ 2 & 68.88 & 61.43 & 19.76 & 24.24 & 11.36 & 14.33 \\ 3 & 71.93 & 77.31 & 18.43 & 14.23 & 9.64 & 8.46 \\ 4 & 78.19 & 80.93 & 14.94 & 13.02 & 6.87 & 6.05 \\ 5 & 81.95 & 84.91 & 12.83 & 11.10 & 5.22 & 3.99 \\ 6 & 86.16 & 87.42 & 10.13 & 9.85 & 3.70 & 2.73 \\ 7 & 89.33 & 88.22 & 7.85 & 9.14 & 2.82 & 2.64 \\ \text { Mean } & 76.79 & 77.03 & 15.58 & 15.47 & 7.63 & 7.50\end{array}$

Conditional on Chronic Health, $\left(H_{i t}=1\right)$. \# Person/Year Obs. $=1,860$

\begin{tabular}{lllllll} 
Mean & 74.65 & 79.27 & 11.84 & 13.64 & 13.51 & 7.09 \\
\hline
\end{tabular}

Table 14: Predicted Transitions

\begin{tabular}{l|lll|lll} 
& \multicolumn{3}{|c|}{ Behavior One Period Post } & \multicolumn{3}{c}{ Behavior Three Periods Post } \\
Behavior One & Not & Light & Heavy & Not & Light & Heavy \\
Period Prior & Smoking & Smoking & Smoking & Smoking & Smoking & Smoking \\
\hline Transitions Around Health Exams & & & & \\
\\
Not Smoking & $98.73 \%$ & $1.22 \%$ & $0.05 \%$ & $97.75 \%$ & $1.98 \%$ & $0.27 \%$ \\
Light Smoking & 11.76 & 63.81 & 24.43 & 21.75 & 54.01 & 24.24 \\
Heavy Smoking & 4.66 & 37.90 & 57.44 & 9.91 & 42.49 & 47.60 \\
Transitions Around Chronic Health Shocks & & & & \\
Not Smoking & 99.16 & 0.50 & 0.34 & 98.92 & 0.65 & 0.43 \\
Light Smoking & 32.78 & 44.09 & 23.13 & 55.86 & 27.55 & 16.59 \\
Heavy Smoking & 11.49 & 34.65 & 53.86 & 24.22 & 27.97 & 47.81 \\
Transitions Around Health Marker Index Shocks: 75 & Percentile & & \\
Not Smoking & 99.04 & 0.93 & 0.03 & 98.18 & 1.65 & 0.17 \\
Light Smoking & 11.66 & 62.62 & 26.22 & 21.33 & 54.94 & 26.73 \\
Heavy Smoking & 4.22 & 33.12 & 62.66 & 8.87 & 37.91 & 53.22 \\
\hline
\end{tabular}


Table 15: Age of Chronic Health Onset and Death

\begin{tabular}{lcc}
\hline \hline Variable & Mean Age of & Mean Age of \\
& Chronic Health Shock & Death \\
\hline Never Smokes & 70.75 & 77.60 \\
Smokes $\leq 1$ Pack/day from Age 18 & $(10.72)$ & $(11.60)$ \\
Smokes $>$ 1 Pack/day from Age 18 & 68.91 & 73.32 \\
& $(10.89)$ & $(11.19)$ \\
Smokes $>$ 1 Pack/day from Age 18 and & 66.79 & 69.58 \\
quits at Age 30 & $(10.87)$ & $(10.94)$ \\
& & \\
quits at Age 40 & 70.77 & 77.58 \\
& $(11.00)$ & $(11.85)$ \\
quits at Age 50 & 70.54 & 77.32 \\
& $(11.33)$ & $(12.28)$ \\
quits at Age 60 & 69.98 & 76.60 \\
& $(11.83)$ & $(11.02)$ \\
& 66.55 & 74.99 \\
& $(10.91)$ & $(13.85)$ \\
\hline
\end{tabular}

Table 16: Posterior Variance by Exam: The Speed of Learning

\begin{tabular}{cccc}
\hline \hline Exam & Mean Posterior Variance & \% Decrease & Cummulative \% Decrease \\
\hline Initial Prior & 0.0095 & - & - \\
1 & 0.0076 & $19.9 \%$ & $19.9 \%$ \\
2 & 0.0069 & $8.7 \%$ & $26.9 \%$ \\
3 & 0.0066 & $4.7 \%$ & $30.3 \%$ \\
4 & 0.0064 & $3.6 \%$ & $32.8 \%$ \\
5 & 0.0061 & $4.0 \%$ & $35.5 \%$ \\
6 & 0.0059 & $3.8 \%$ & $37.9 \%$ \\
7 & 0.0057 & $2.9 \%$ & $39.7 \%$ \\
\hline
\end{tabular}

